\title{
Identifying dramatic selection shifts in phylogenetic trees
} Karin S Dorman*

Address: Departments of Statistics and Genetics Development \& Cell Biology, and the Program in Bioinformatics and Computational Biology, Iowa State University, Ames, IA, USA

Email: Karin S Dorman* - kdorman@iastate.edu

* Corresponding author

from First International Conference on Phylogenomics

Sainte-Adèle, Québec, Canada. 15-19 March, 2006

Published: 8 February 2007

BMC Evolutionary Biology 2007, 7(SuppI I):SI0 doi:I0.II86/I47I-2I48-7-SI-SIO

(c) 2007 Dorman; licensee BioMed Central Ltd.

This is an Open Access article distributed under the terms of the Creative Commons Attribution License (http://creativecommons.org/licenses/by/2.0), which permits unrestricted use, distribution, and reproduction in any medium, provided the original work is properly cited.

\begin{abstract}
Background: The rate of evolution varies spatially along genomes and temporally in time. The presence of evolutionary rate variation is an informative signal that often marks functional regions of genomes and historical selection events. There exist many tests for temporal rate variation, or heterotachy, that start by partitioning sampled sequences into two or more groups and testing rate homogeneity among the groups. I develop a Bayesian method to infer phylogenetic trees with a divergence point, or dramatic temporal shifts in selection pressure that affect many nucleotide sites simultaneously, located at an unknown position in the tree.

Results: Simulation demonstrates that the method is most able to detect divergence points when rate variation and the number of affected sites is high, but not beyond biologically relevant values. The method is applied to two viral data sets. A divergence point is identified separating the B and C subtypes, two genetically distinct variants of HIV that have spread into different human populations with the AIDS epidemic. In contrast, no strong signal of temporal rate variation is found in a sample of $\mathrm{F}$ and $\mathrm{H}$ genotypes, two genetic variants of $\mathrm{HBV}$ that have likely evolved with humans during their immigration and expansion into the Americas.

Conclusion: Temporal shifts in evolutionary rate of sufficient magnitude are detectable in the history of sampled sequences. The ability to detect such divergence points without the need to specify a prior hypothesis about the location or timing of the divergence point should help scientists identify historically important selection events and decipher mechanisms of evolution.
\end{abstract}

\section{Background}

The rate of evolution at a site at one moment in time depends on the underlying mutation rate and the overlying selective constraints. Both determinants of evolutionary rate may change spatially, along the genome, or temporally, in evolutionary time, to produce evolutionary rate variation. Many highly important biological processes manifest in sequence data as spatial or temporal rate variation, and this signal is often harnessed to extract biological information from sampled sequences. Sites in a gene with high nonsynonymous rates may be responding to positive selection (e.g. [1-3]), while conserved genomic sites likely carry out mission-critical biological functions (e.g. $[4,5])$. Temporal shifts in evolutionary rate suggest functional change and may be used to explain the evolution of novel traits (e.g. $[6,7])$, to characterize functional 
innovation within gene families (e.g. [8-11]), and to resolve phylogenetic discrepancies (e.g. $[12,13])$.

As soon as molecular sequence data were available, evolutionary rates were observed to vary among sites. Some amino acid positions seem completely invariant in proteins [14], and a nucleotide model with an unknown fraction of invariant sites better approximates mitochondrial data [15]. This two-class, variable or invariant, site classification can generalize to any discrete distribution of rates across site [16]. Theory and simulations suggest that phylogenetic inference is particularly sensitive to unrecognized site-to-site rate variation $[17,18]$. Models that incorporate spatial rate variation often fit biological sequence data statistically better than models that assume a constant rate [19-24]. Probably the most common model for site-to-site rate variation is the discrete approximation to gamma distributed rates [25], but all these models are collectively referred to as RAS models for rates across sites.

Temporal rate variation, also known as heterotachy, is not so easy to observe directly, but it is a long-standing idea $[26,27]$. Early work produced the word covarion, concomitantly variable codon, also extended to nucleotides [28], to name the concept of temporal rate variation. In the original covarion model an approximately constant fraction of sites evolve, accumulating variation, but the site membership in the variable pool is continuously changing in time. The covarion model experienced very little theoretical progress until formalized as a Markov model in 1998 [29]. Then, phylogenetic implementations followed rapidly $[30,31]$. Recently, the covarion model was extended to allow switching between more than two site classes [32]. Heterotachy models have largely assumed the switching rate is constant throughout time.

There is increasing evidence that heterotachy is an important evolutionary phenomenon, perhaps exceeding or superseding the importance of site-to-site rate variation $[33,34]$. Much of this evidence is generated by testing the hypothesis of equal rates between pre-defined clades, for which many tests have been derived [10,11,31,33,35-40]. An alternative strategy attempts to detect brief spurts of evolution presumed to occur coincident with functional innovation. Such episodic evolution leaves signatures on branches of phylogenetic trees, where the ratio of nonsynonymous to synonymous substitution rates exceeds one [41-43]. Application of both kinds of techniques to large data sets reveals widespread heterotachy $[44,45]$. These tests are undoubtedly most sensitive to dramatic shifts in selection pressure, where many sites simultaneously experience an altered evolutionary rate. Gene duplication, environmental changes, and niche invasion are all associated with large-scale changes in selection pressure affect- ing many sites in a genome. There are two questions of interest: (1) whether selection shifts occur, for example after gene duplication, and (2) when selection shifts occur in the history of sequences, e.g. to time historical niche invasion based on a sample of extant species. Henceforth, we shall call the locations of these shifts divergence points in time or phylogenetic trees.

Gu [36] describes a maximum likelihood method for detecting divergence points associated with gene duplication. Given a set of homologous genes categorized into paralogous groups separated by gene duplication and the phylogenetic trees that relate orthologs within groups, the method can identify amino acid positions that are functionally divergent between the groups. It works by hypothesizing that a fraction $\theta$ of amino acid sites (divergent sites) acquire independent function and thereby independent evolutionary rates in two or more of the ortholog groups. The remaining constrained sites retain function and evolve at the same dependent rate in all groups. After maximum likelihood estimation of $\theta$, subtree branch lengths, and evolutionary parameters, Bayes rule can predict functionally important residues if the estimate $\hat{\theta}$ is significantly bigger than zero.

The present paper extends the Gu method by performing inference on a full tree without specifying a priori the branch where a divergence point is expected. I develop the method in a Bayesian context for nucleotide sequences and test it using a panel of simulated sequences. I then apply the method to study divergence between Human Immunodeficiency Virus (HIV) subtypes and Hepatitis B Virus (HBV) genotypes, revealing a substantial selection event sometime after separation of HIV subtypes B and C and no evidence of a selection event separating HBV genotypes $\mathrm{F}$ and $\mathrm{H}$.

\section{Results and Discussion Simulation}

Varying the magnitude of the selection shift

I simulate data sets assuming a single divergence point in a topology relating eight hypothetical taxa (Figure 1). I use a discrete gamma distribution with four rate categories and the same shape parameter $\alpha$ to approximate the variation in both spatial and temporal rates [25]. To explore the sensitivity of the method to the strength of the temporal selection shift, I vary the degree of rate variation (smaller $\alpha$ implies greater rate variation) and the fraction of sites $\theta$ experiencing a selection shift across the divergence point. When a site is selected to shift rate, it randomly selects a new rate class from the discrete gamma distribution. All other parameters do not vary in this first set of simulations. In particular, the simulated divergence 
point is located at relative position $l=0.9$ on branch $b=8$ in the topology $\tau$ of Figure 1 with $t_{j}=0.1$ expected mutations (transition/transversion ratio $\kappa=2$ ) per site along each branch $j=1, \ldots, 13$. C code implementing Markov chain Monte Carlo sampling of the posterior distribution analyzes each simulated alignment. Posterior statistics of model parameters are computed along with the Bayes factor $B_{D P}$ in favor of a divergence point somewhere in the tree. When $\log _{10} B_{D P}>1$, there is strong support for a divergence point, which then allows conditional estimation of $\theta_{1} l$, and the Bayes factor $B_{j}$ favoring a divergence point located specifically on branch $j$. All these latter statistics are based on the subset of MCMC samples that have a divergence point.

Figure 2 plots the method type I error rate and power for the various simulation conditions (blue bars) when the null hypothesis is homotachy. Here, type I errors result when there is no divergence point, but the user concludes one because $\log _{10} B_{D P}>1$. Type I errors do not occur for any of the 500 simulations without a divergence point. Power is the probability that the method strongly sup-

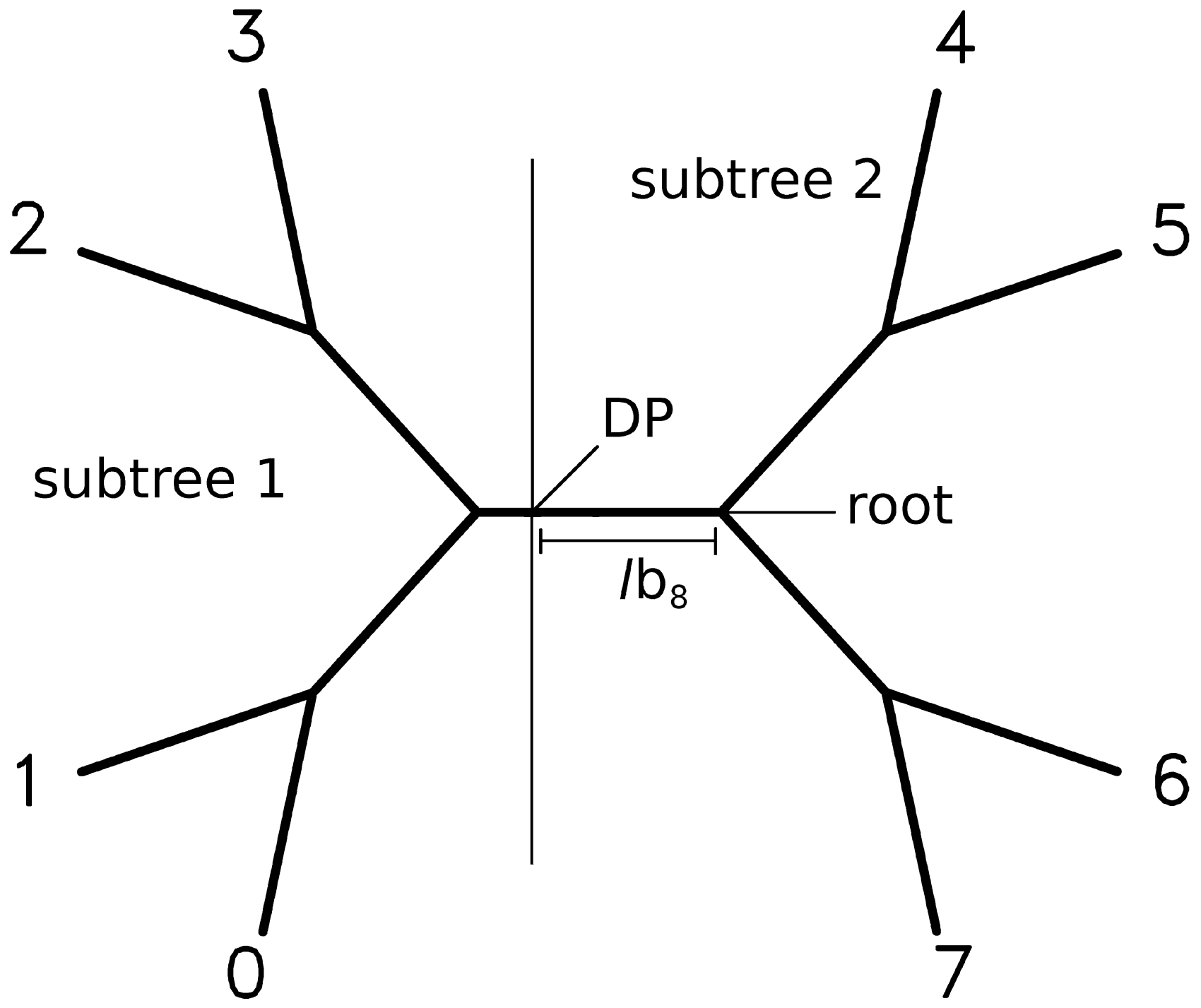

Figure I

Simulation tree. The phylogenetic tree used for simulation. There are eight taxa, labeled 0 to 7 , related according to the depicted topology with all branch lengths equal. Each simulation assumes a single divergence point (DP) located at a distance $I$ $=0.9$ away from the right end of the middle branch, known as branch 8 . The vertical line, splits the full phylogeny into two subtrees. Subtree 2 has a stubbed branch where it connected to subtree I of length $I t_{8}$. Subtree I has a stubbed branch of length $(I-I) t_{8}$. 
ports a divergence point when one is simulated. For frequentist methods, the type II error rate is one minus the power, i.e. the probability of accepting the null hypothesis when the alternative hypothesis of heterotachy is actually true. Bayesian analyses are advantageous when it comes to assessing the strength of the null hypothesis. In this case, one should not commit to the null hypothesis of homotachy unless it receives strong support, e.g. $\log _{10} B_{D P}<-1$, which here occurs for only nine of 2500 datasets simulated with a divergence point and only when $\theta=0.1$. A more important concern for the Bayesian method is the decreasing power to detect the divergence point as rate variation and the fraction of sites subject to rate shifts decrease. When $\theta=0.1$, the divergence point becomes effectively undetectable. For all other simulated values of $\theta$, the divergence point is detectable given sufficient rate variation. When $\alpha=2$, the method never works well, and the rates for the four discrete categories are $0.3,0.7,1.1$ and 2.0, yielding less than seven-fold differences in rate. Susko et al. [11] use a regression technique to estimate the size of rate differences between eukaryotic and archaebacterial amino acid sequences of elongation factor $1 \alpha$ and find rate variation roughly between 3 and 15-fold, just straddling the level of rate variation detectable in this simulation.

When a simulated divergence point is highly supported, the identification of the branch with the divergence point is exceptionally successful via Bayes factor $B_{j}$ for branch $j$. Out of 1195 simulations with high support for the divergence point, only 4 failed to also identify the true branch 8 as highly likely $\left(\log _{10} B_{8}>1\right)$ to carry that divergence point. Only twice, another branch is incorrectly found to strongly favor a divergence point somewhere along its length. These results demonstrate the method can not only detect the presence of a divergence point in a phylogenetic tree, but also pinpoint the affected branch with high confidence.

Table 1 records the posterior mean (indicating accuracy) and width of the 95\% Bayesian credible intervals (indicating precision) for parameters $\alpha, \theta$ and $l$ averaged across simulated data sets. Figure 3 plots the distributions of posterior means for these parameters as well as $\kappa$ and two branch lengths: $t_{8}$ is the length of the branch carrying the divergence point and $t_{12}$ is that of a randomly selected terminal branch. Each entry in Table 1 and boxplot in Figure 3 is based on 100 estimated values except those for $\theta$ and $l$, which may be estimated in far fewer simulations. Estimates of $\alpha$, which are logged before plotting in Figure $3(\mathrm{a})$, tend to overestimate the true value, especially when true $\alpha=0.01$ and as the fraction of heterotachous sites increases. Evolutionary rate parameter $\kappa$, the transition/ transversion ratio, is fairly well estimated but with a slight upward bias when site-to-site rate variation is high $(\alpha<$
$0.5)$. In contrast, estimation of $\theta$ is poor. While there is relatively low posterior uncertainty in $\theta$ (as compared to $l$ ), the estimates are dramatically and increasingly downward biased as true $\theta$ climbs above 0.1 . The effect is not just a consequence of the prior, which would tend to pull estimates toward the prior mean of 0.5 , because even for true $\theta \leq 0.5$, the bias is downward. The bias is most noticeable for those datasets that detect the divergence point. When simulating $\theta=0.9$ and $\alpha=0.01$, the divergence point is always detected with high confidence, but the 95\% Bayesian credible intervals for $\theta$ never contain the true value. For estimating the divergence point location $l$, the fact that the estimates pull toward the true value 0.9 as heterotachy increases suggests that there is some information in the data about this parameter, however the information is weak as demonstrated by the very wide Bayesian credible intervals in Table 1. Given this result, a model that simply places divergence points at internal nodes of the tree may have just as much power to detect divergence events, while simplifying the MCMC algorithm and convergence. Finally, estimates of all branch lengths tend to be less precise with increasing site-to-site rate variation. In addition, branch 12 is increasingly overestimated as the amount of noticeable heterotachy increases. Since temporal and spatial rate variation can be somewhat or completely confounded [29], it is not surprising to find estimation of $\alpha$ and $\theta$ somewhat entangled. Additionally, failure to adequately account for site-to-site rate variation, in this case because $\alpha$ is overestimated, is known to produce biased branch length estimates [46].

Whether the performance in simulation translates to real biological sequences is still questionable. Previous analyses suggest that biological site-to-site rate variation falls in the range $\alpha \in(0.1,10)$, with nonsynonymous (and more likely selected) rate variation tending to fall below $\alpha=1$ $[24,46,47]$. While there is less information about biologically relevant ranges for $\theta, \mathrm{Gu}$ [36] estimates $\theta=0.46$ for a study of the cyclooxygenase gene family at the amino acid level. Comparison of rates between pre-defined monophyletic groups shows very high proportions of sites eventually experience heterotachy during evolution, even in functionally conserved sequences, for example, $66 \%$ of rRNA sites [48] or as high as $47 \%$ of the cytochrome $b$ amino acids [49]. Yang and Nielsen [42] find the proportion of codons undergoing positive selection during episodic evolution along particular lineages to be between 0.03 and 0.2 , depending on the gene analyzed. Thus, it appears that the power of this method to detect divergence points may falter near the boundary of biological relevance. In the next section, additional simulations investigate the amount of information, as measured by the sequence divergence, alignment length, and number of taxa, needed to detect divergence points. 

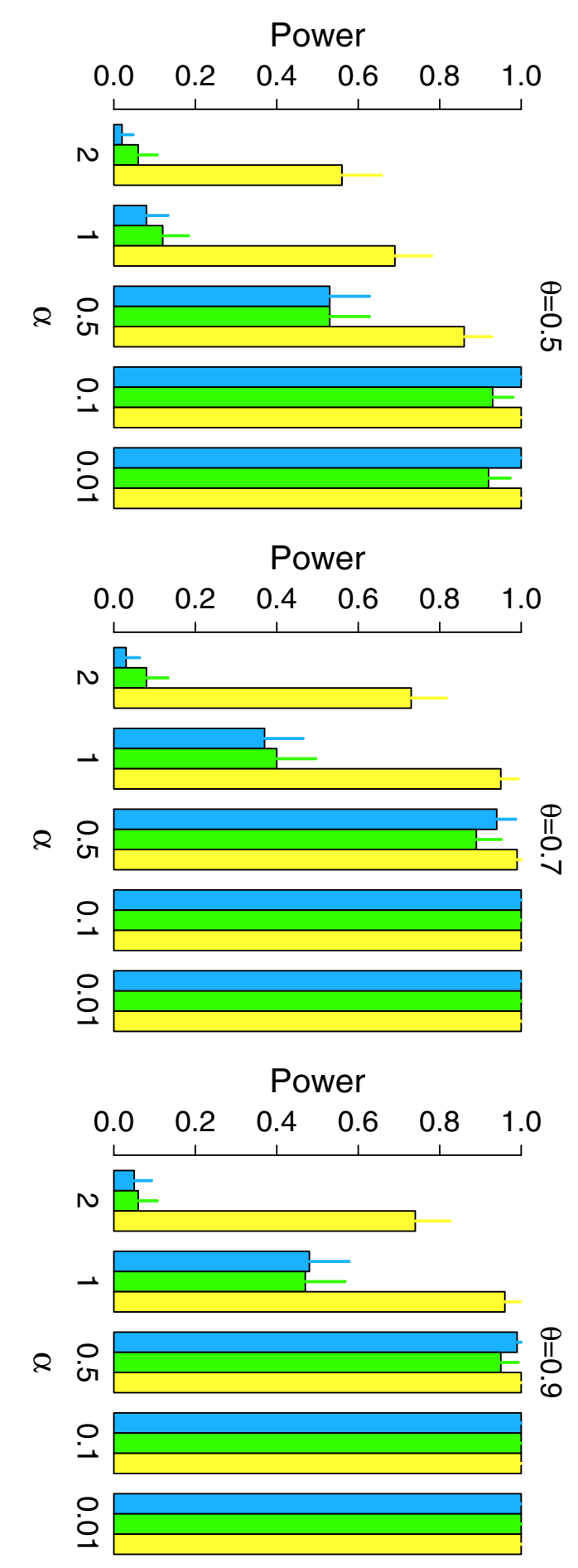
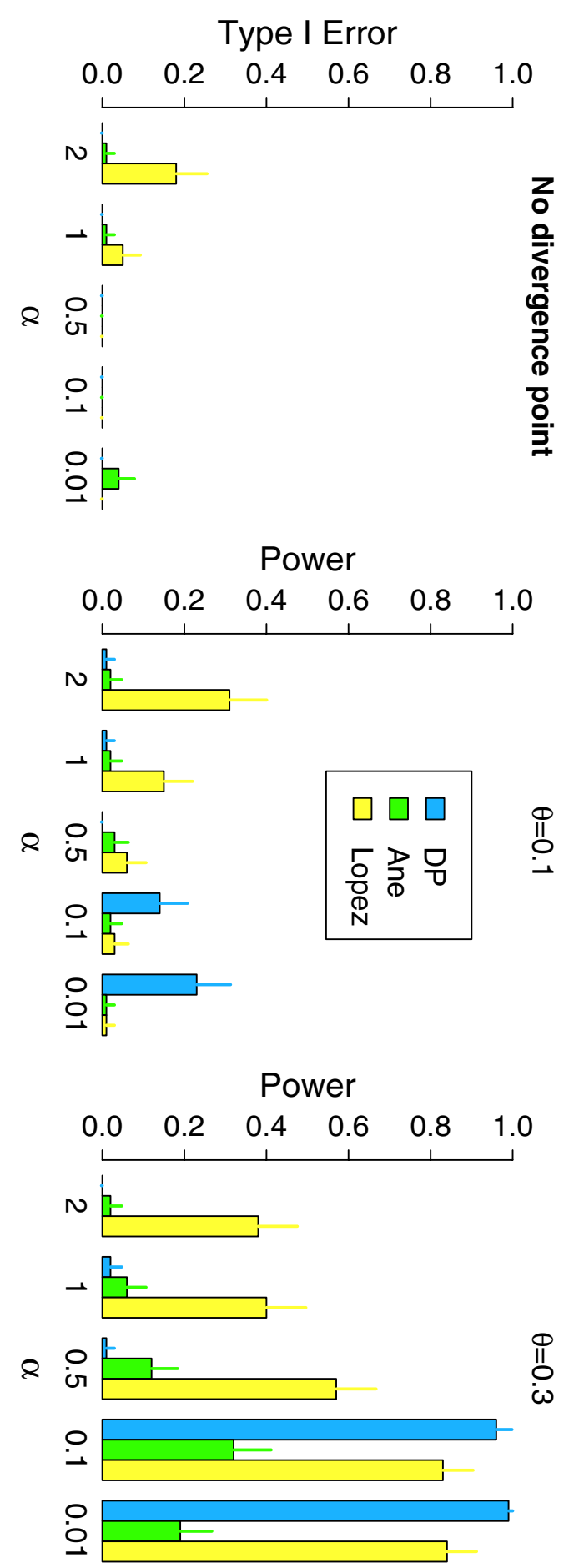

\section{Figure 2}

Method comparison. The type I error rate and power of the new method are compared with two other methods, that of Ané et al. [33] and Lopez et al. [13]. The null hypothesis is no temporal rate variation or homotachy. Type I error means the method rejects the null when it is actually true. Power is the probability the method correctly rejects the null when there actually is a divergence point. For the Bayesian method, the null hypothesis is rejected if $\log _{10} B_{D P}>1$. Error bars indicate $95 \%$ confidence intervals accounting for simulation error. 


\section{Varying the amount of data and evolution}

To explore the power of the method to detect the divergence point for varying amounts and diversity of input data, I generate simulated data sets under a variety of conditions. I start by again simulating data alignments using the tree of Figure 1. For these simulations, I set $\alpha=0.7$ and $\theta=0.5$ and vary both the branch length (all branches of the topology are equal) and the length of the alignment. Figure 4 displays the results, showing that increasing diversity, as measured by the branch length, and data, as measured by the alignment length, both improve the power of the method to detect the divergence point. In particular, the divergence point is detectable for these $\alpha$ and $\theta$ when the branch length is above 0.07 and the alignment length is over 5000. Similar patterns are observed for different $\alpha$ and $\theta$ combinations. As long as $\theta \geq 0.3$, the method achieves good power at least for the simulation with $t_{j}=0.9$ and 7500 base pairs (data not shown).

To test the impact of including more sequences, I simulate data with increasing numbers of taxa in each subtree. This time, $\alpha=0.5, \theta=0.5$, and all branch lengths $t_{j}=0.1$ are selected to demonstrate a range of outcomes in resulting power. The original simulation tree of Figure 1 has 8 taxa. I also simulate sequences with 4,12 , or 16 taxa, maintaining the divergence point on the middle branch and adding taxa in a balanced fashion to both subtrees. Because all branch lengths are held constant, any effect of adding more taxa could be a consequence of the additional taxa or the increase in total evolutionary time simulated. The power of the method to detect the divergence point increases substantially with the number of taxa in each subtree (Figure 5). Unfortunately, the computational cost also increases substantially. Roughly, based on informal observation only, 8 taxa take ten times as long as 4 taxa, and computational times double for every 4 additional taxa after that. Finally, I also examine the probability of detecting heterotachy when the divergence point is placed on a terminal branch rather than the internal branch of Figure 1 . This time $\alpha=\theta=t_{j}=0.1$. Not surprisingly, power of the method to detect a terminal branch divergence point is substantially compromised (Figure 5), indicating that balanced subtrees including many taxa provide the ideal conditions for detecting a divergence point.

\section{Comparison to existing heterotachy detection methods}

Figure 2 compares the power of the Bayesian divergence point method with two other statistical tests for heterotachy given pre-defined subgroups. Ané et al. [33] recently describe a parametric bootstrap test of the covarion model that tests the degree of independence in the proportion of invariant sites in the two subgroups. When applied to the first set of simulated data, this method demonstrates a low type I error rate in the absence of heterotachy and comparable power to the Bayesian method in the presence of heterotachy except when $\theta=0.1$ and $\alpha$ is small. However, the method is not ideally matched to the simulations since it specifically tests the covarion model with invariant sites, but the simulation model allows no truly invariant sites. A more appropriate test is suggested by Lopez et al. [49], who describe a method to compare the number of substitutions in each subgroup at each site. Under the homotachous model, the number of substitutions at a site should be proportional to the amount of evolution, or tree length, of each subtree. Substantial deviations from this expectation, as measured by a chi-square statistic, indicate a change in evolutionary rate between the two subtrees. As expected, this method has more power than

Table I: Estimation of $\alpha, \theta$, and $I$.

\begin{tabular}{|c|c|c|c|c|c|c|c|c|c|c|c|c|c|c|c|}
\hline \multirow[b]{2}{*}{$\theta \alpha$} & \multicolumn{5}{|c|}{ Estimation of $\alpha$} & \multicolumn{5}{|c|}{ Estimation of $\theta$} & \multicolumn{5}{|c|}{ Estimation of $I$} \\
\hline & 2.0 & 1.0 & 0.5 & 0.1 & 0.01 & 2.0 & 1.0 & 0.5 & 0.1 & 0.01 & 2.0 & 1.0 & 0.5 & 0.1 & 0.01 \\
\hline \multirow[t]{2}{*}{0.0} & 2.05 & 0.99 & 0.49 & 0.10 & 0.04 & NA & NA & NA & NA & NA & NA & NA & NA & NA & NA \\
\hline & 1.90 & 0.61 & 0.23 & 0.05 & 0.07 & NA & NA & NA & NA & NA & NA & NA & NA & NA & NA \\
\hline \multirow[t]{2}{*}{0.1} & 2.18 & 1.07 & 0.54 & 0.11 & 0.05 & 0.76 & 0.68 & NA & 0.17 & 0.15 & 0.55 & 0.58 & NA & 0.51 & 0.51 \\
\hline & 2.14 & 0.69 & 0.28 & 0.08 & 0.08 & 0.99 & 0.96 & NA & 0.21 & 0.17 & 0.97 & 0.99 & NA & 0.97 & 0.97 \\
\hline \multirow[t]{2}{*}{0.3} & 2.77 & 1.23 & 0.59 & 0.11 & 0.05 & NA & 0.49 & 0.38 & 0.26 & 0.26 & NA & 0.53 & 0.57 & 0.58 & 0.57 \\
\hline & 3.42 & 0.91 & 0.36 & 0.08 & 0.08 & NA & 0.55 & 0.48 & 0.22 & 0.19 & NA & 0.97 & 0.97 & 0.96 & 0.97 \\
\hline \multirow[t]{2}{*}{0.5} & 3.27 & $1.4 \mid$ & 0.60 & 0.11 & 0.06 & 0.79 & 0.56 & 0.43 & 0.40 & 0.40 & 0.37 & 0.56 & 0.57 & 0.63 & 0.61 \\
\hline & 4.59 & 1.21 & 0.40 & 0.09 & 0.10 & 0.85 & 0.73 & 0.47 & 0.23 & 0.21 & 0.88 & 0.99 & 0.96 & 0.96 & 0.95 \\
\hline \multirow[t]{2}{*}{0.7} & 4.87 & 1.45 & 0.62 & 0.12 & 0.08 & 0.80 & 0.62 & 0.52 & 0.55 & 0.54 & 0.50 & 0.53 & 0.58 & 0.66 & 0.65 \\
\hline & 7.85 & 1.49 & 0.40 & 0.08 & 0.10 & 0.89 & 0.74 & 0.50 & 0.24 & 0.22 & 0.95 & 0.96 & 0.95 & 0.94 & 0.94 \\
\hline \multirow[t]{2}{*}{0.9} & 6.74 & 1.62 & 0.65 & 0.13 & 0.10 & 0.86 & 0.74 & 0.66 & 0.69 & 0.69 & 0.47 & 0.56 & 0.61 & 0.67 & 0.66 \\
\hline & II.44 & 1.83 & 0.40 & 0.08 & 0.10 & 0.86 & 0.72 & 0.48 & 0.24 & 0.23 & 0.92 & 0.97 & 0.94 & 0.94 & 0.93 \\
\hline
\end{tabular}

Each pair of entries summarizes the posterior estimation of model parameters $\alpha, \theta$, and $I$ from 100 random data sets simulated assuming various choices of $\theta$ (rows) and $\alpha$ (columns). The first row, where $\theta=0$ is for data simulated without a divergence point. First in each pair is the average posterior mean, summarizing accuracy; second is the average width of the $95 \%$ Bayesian credible interval, summarizing precision. Statistics for $\theta$ and $I$ are based only on those simulations strongly supporting a divergence point, which may be substantially fewer than 100 simulations. NA means a divergence point was never strongly supported for that simulation condition. 
(a)

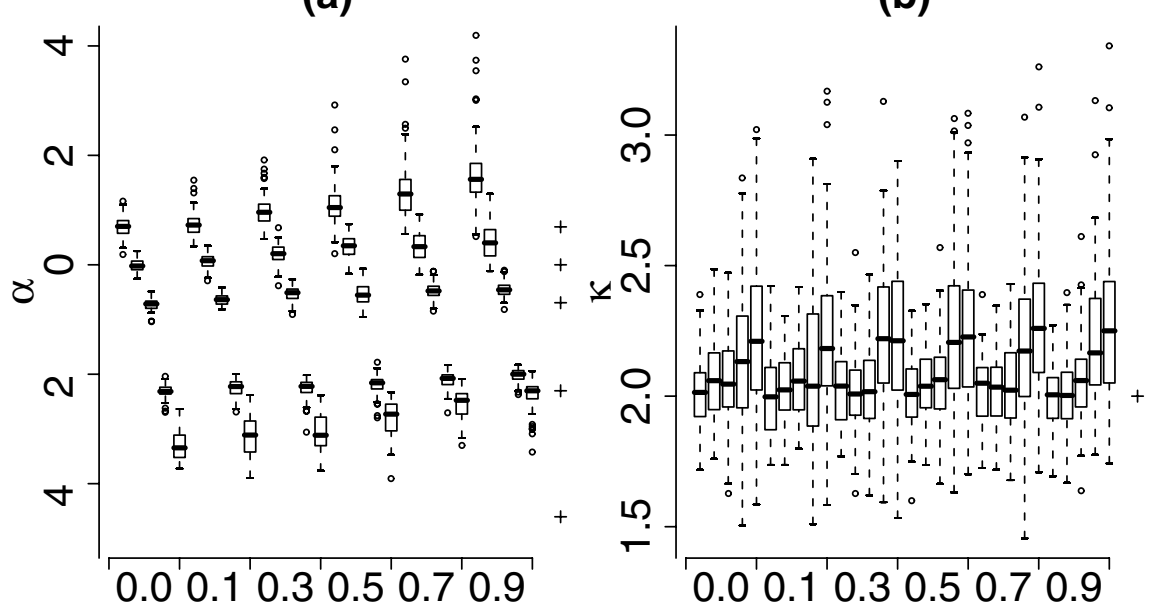

(d)

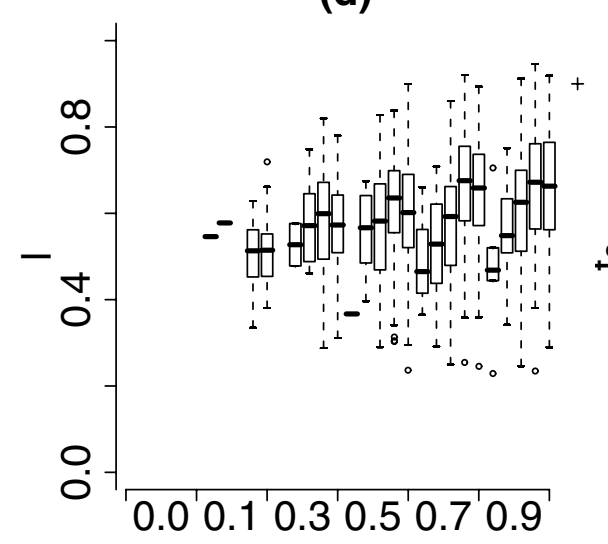

(e)

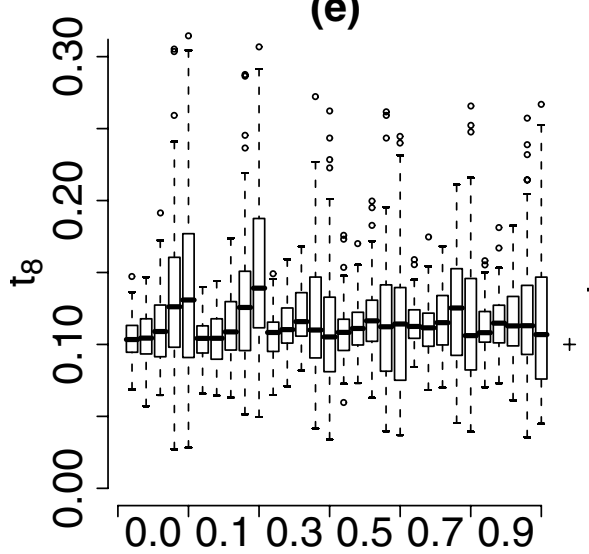

(c)

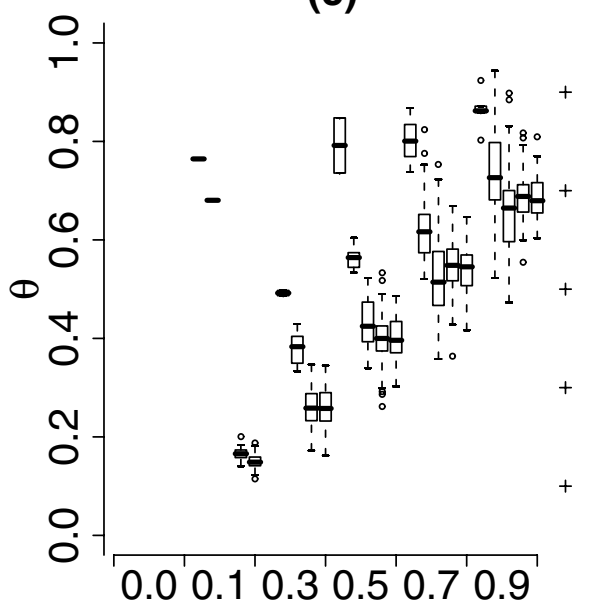

(f)

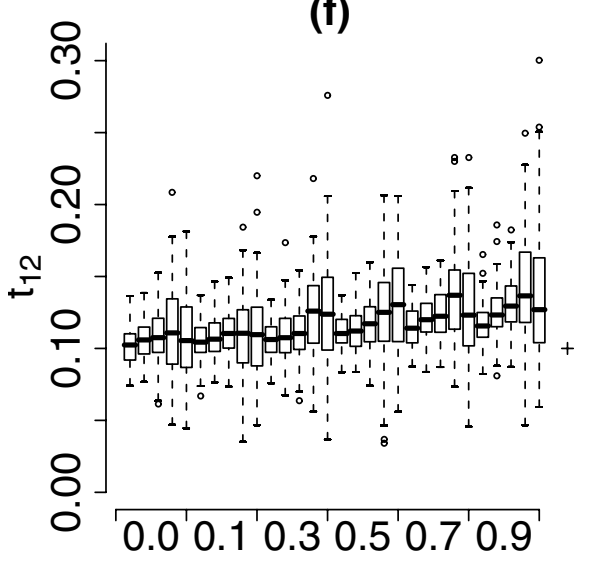

$$
\theta[\alpha=0.01,0.1,0.5,1.0,2.0]
$$

Figure 3

Parameter estimation. Boxplots of posterior mean estimates of (a) $\alpha$, (b) $\kappa$ (c) $\theta$, (d) $I$, (e) branch length of the 8th branch $t_{8}$ (the one with the divergence point), and ( $f$ branch length of the 12 th branch $t_{12}$. Each boxplot is based on 100 simulations, except (c) and (d), where posterior means of $\theta$ and $I$ are only estimated for those simulations strongly supporting the divergence point. Results are grouped by simulated $\theta$ value as marked on the x-axis. There are five simulations per group, with simulated $\alpha$ decreasing 2.0, I.0, 0.5, 0.1, 0.0 I. The arrangement is such that temporal rate variation is generally increasing from left to right. The estimates of $\alpha$ are logged before plotting to better show the variation in the smaller values. The location of the true value(s) of each parameter are marked by a + just right of its plot.

the Ané et al. and also beats the Bayesian method. In particular, it is better able to detect heterotachy when $\alpha>0.5$ and there is low site-to-site rate variation. However, these conditions are also the ones where the method's type I error rate begins to exceed expectation (see Figure 2, No divergence point and $\theta=0.1$ ). Thus, it may be that the conservative behavior of both the Ané et al. and Bayesian methods in the presence of low rate variation are desirable.

\section{HIV}

As HIV spread into the human population in the last century, genetically distinct lineages arose [50]. These socalled subtypes have distinct geographic distributions [51]. In particular, subtype B dominates throughout much of the non-African and non-Asian world, while subtype $\mathrm{C}$ dominates in southern and eastern Africa, parts of the Middle East, and India [51]. Much of the geographic restriction of subtypes can be explained by the travels of a 


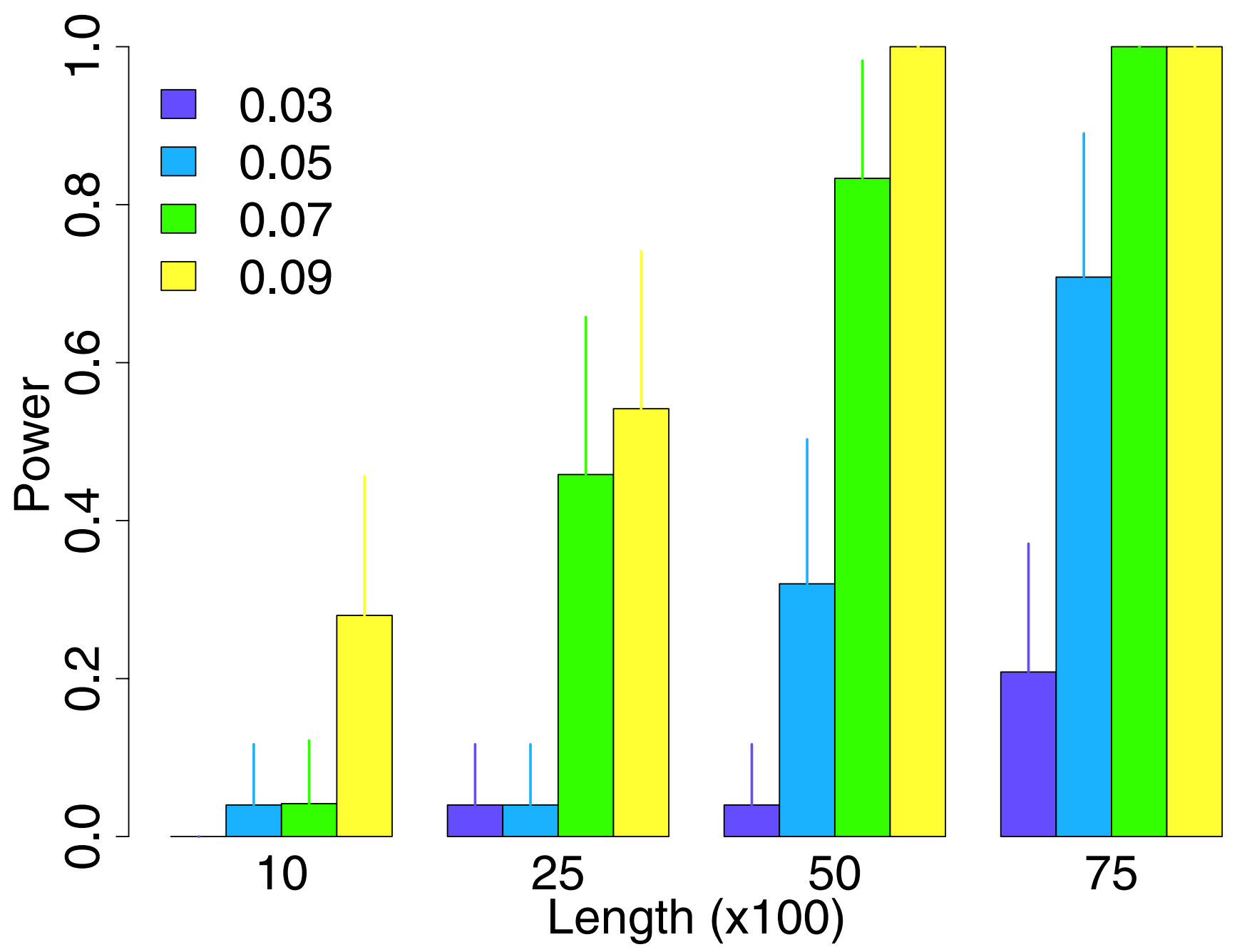

Figure 4

Sample size and power. The proportion of simulations that strongly support the simulated divergence point when $\alpha=0.7$ and $\theta=0.5$. Each group of bars corresponds to a different alignment length ranging through 1000, 2500, 5000, and 7500 . Within the group, there are four different branch lengths assigned to every branch in the simulation topology of Figure I, either $0.03,0.05,0.07$, or 0.09 . Error bars indicate $95 \%$ confidence intervals accounting for simulation error.

few infected individuals [52], however there is also evidence of population level selection on the virus, particularly in relation to immune selection [53]. I hypothesize that if the virus encounters substantial population-specific selection pressures when entering a new population, a selection shift signature may be detectable on the branches of phylogenetic trees that separate subtypes.

To test the hypothesis, I align 10 HIV sequences, five from subtype B and five from subtype C. Summaries of the marginal posterior distributions for each continuous parameter of the model are shown in Table 2. The reported potential scale reduction factors [54] demonstrate healthy agreement between the six independent MCMC runs and all six runs are combined for statistical estimation. The Bayes factor in favor of a divergence point cannot be computed because the support for a divergence point is unanimous in the posterior sample. The model clearly identifies a highly supported divergence point on the branch separating subtypes $B$ and $C$, with $\log _{10} B_{B C}=4.08$, where indexing is meant to indicate the branch separating $\mathrm{B}$ and C. Figure 6 shows the location of the estimated divergence point along with its 95\% Bayesian credible interval on the phylogeny drawn with branch lengths at their posterior means. The precise location of the divergence point along the branch is poorly estimated, but the selected branch is highly supported. Considering the esti- 

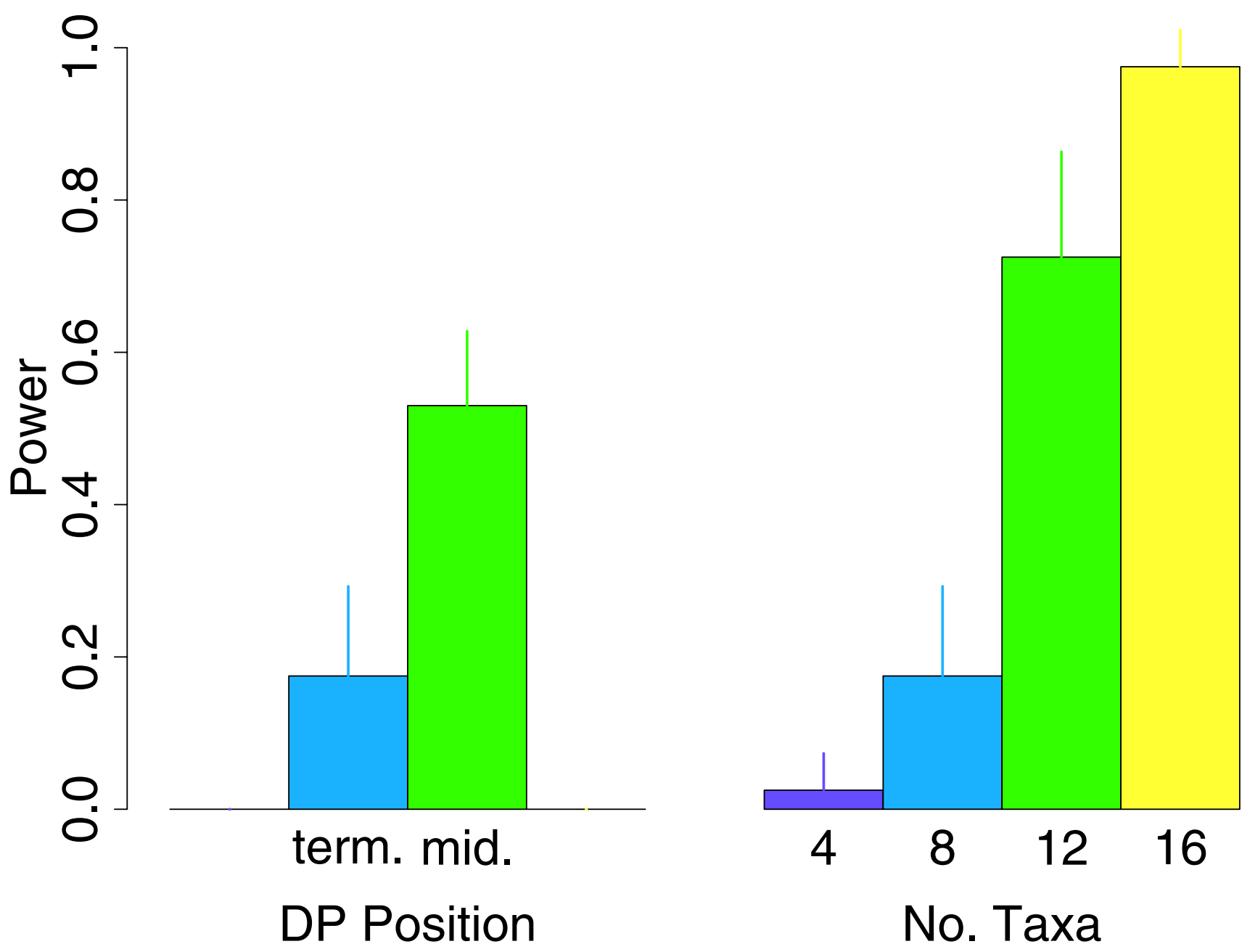

Figure 5

Power as a function of group size. The power of the method to detect the divergence point with strong support as the branch location of the divergence point or the size of the topology changes. For the first set, the divergence point is located on the middle branch or a terminal branch of the 8-taxa tree of Figure I and $\alpha=0.5, \theta=0.5, t_{j}=0.1$, and $L=1000$. For the second set, the divergence point is located on the middle branch of a 4-, 8-, I2-, or 16-taxa tree, and $\alpha=0.1, \theta=0.1, t_{j}=0.1$, and $L=$ 1000. Error bars indicate $95 \%$ confidence intervals accounting for simulation error.

mated values of $\alpha=0.23$ and $\theta=0.28$, the alignment length $L=6610$, and the average branch length ( $\bar{t}=0.04$ ) of this data set, simulation results (not shown) suggest that the method just has enough power to detect the presence of a divergence point. It may not be possible to detect heterotachy for shorter regions of HIV.

\section{HBV}

Like HIV, HBV has diverged into genetically distinct lineages with nonuniform geographic distribution around the world [55]. In the case of HBV, these lineages are called genotypes. Although the origins of HBV are unclear, HBV is most likely to have evolved with humans since our emigration from Africa [56]. The genotypes and their geo- graphic distribution can thus be associated with major migration events, but it remains unclear whether the genotypes express distinct disease phenotypes [57-59]. HBV genotypes $\mathrm{F}$ and $\mathrm{H}$ are restricted to the Americas, probably arriving on these continents with the first human immigrants [57]. Genotype $\mathrm{H}$ is found much less frequently than $F$, and its origin is uncertain [60]. In fact, its classification as a separate genotype is controversial [61]. Given the best estimate of HBV origins, it is not likely that the spread of HBV into new human populations has exerted recent selective pressure on the virus, however co-evolution of the virus along with the human host may create divergence points along branches where humans and viruses co-adapted to new ecological niches. 
Table 2: HIV estimated parameters.

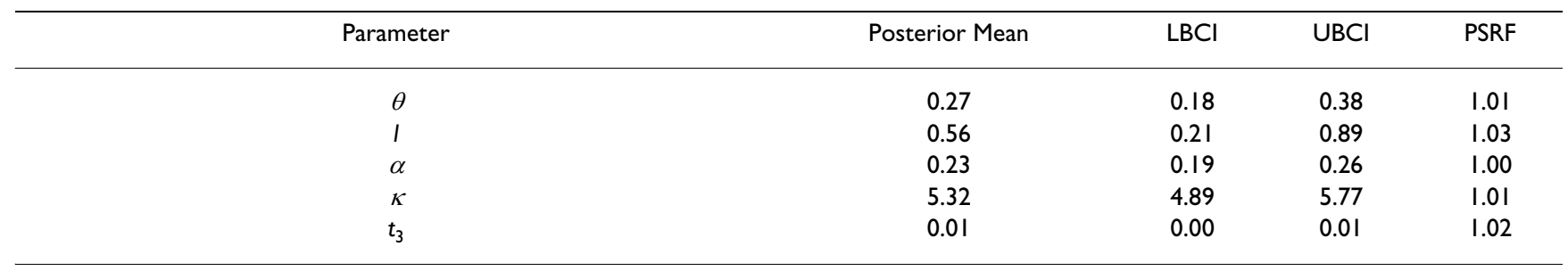

$\log _{10} B_{D P} \approx \inf$ (decisive support for)

$\log _{10} B_{B C}=4.08$ (decisive support for)

The posterior mean, upper and lower 95\% Bayesian credible interval bounds ( $\mathrm{UBCl}$ and $\mathrm{LBCl}$ ) and potential scale reduction factor (PSRF) [54] for all continuous parameters of the model. Data for the branch length with largest PSRF is reported. The last two rows report the Bayes factor in support of a divergence point and the Bayes factor in support of a divergence point along the middle branch.

To look for divergence points related to the emergence of HBV genotypes $\mathrm{F}$ and $\mathrm{H}$, I align seven genotype $\mathrm{F}$ sequences and three genotype $\mathrm{H}$ sequences. Posterior summaries are in Table 3. Figure 7 displays the estimated phylogeny relating these 10 sequences with the branch lengths drawn proportional to their posterior means. The number accompanying each branch is the conditional posterior probability that the divergence point lies somewhere along that branch given one exists somewhere in the tree. In contrast to the HIV results, a divergence point is not supported by the data with $\log _{10} B_{D P}=-0.64$. Considering only the posterior sample supporting a divergence point (1123 samples), no branch shows evidence of strong heterotachy, although the posterior distribution across branches is significantly different from the uniform prior ( $p$-value $<0.001$ ). The alternative hypothesis of homotachy is substantially, but not strongly supported, and the method may simply have insufficient power to detect heterotachy in this data set. Notably, because H is a poorly sampled genotype, the three representatives included here are highly similar, thereby forcing any potential genotype-associated divergence point onto what is effectively a terminal branch. Table 4 suggests power is low under this condition, but I performed no simulations with parameters matching the HBV data, so it is unclear whether the method should have sufficient power to estimate the presence of a divergence point. Evidence of siteto-site rate variation is high, with the posterior mean $\alpha=$ 0.04 , however the low diversity (average branch length $0.015)$ and short alignment (3,215 base pairs) sharply reduce the power of the method.

In addition, strong spatial rate variation may not translate to strong temporal rate variation in the case of HBV. Normally, the magnitude of temporal rate variation is expected to approximately match the magnitude of spatial rate variation, because choosing a new function for a site is roughly equivalent to selecting a new site at random from the same protein $[29,62]$. The model makes this assumption by using the same rate class distribution for spatial and temporal rate variation. Strong purifying selection combined with an error-prone reverse transcriptase is expected to produce highly heterogeneous rates in HBV, with widespread conservation due to overlapping reading frames interrupted by a limited number of mutation-tolerant sites [63]. But for a dual-coding nucleotide to temporally shift rate class, it must acquire a new function in both reading frames. This dual constraint may eliminate the possibility of divergence points in HBV and certainly reduces both the magnitude of temporal rate shifts and the number of affected sites. In short, the biology of HBV may limit both the presence of and the power to detect divergence points. Increasing the number of sampled sequences per genotype may restore power, but this option is not examined further here.

\section{Conclusion}

Spatial and temporal rate variation is the signature left on genomic sequences by the force of selection. Although mechanistic differences can also generate evolutionary rate variation, it appears that the selection signal is stronger [24]. A flurry of new methods have emerged to detect and utilize this signal to inform on function $[43,64,65]$. I propose a new method for inferring the location of divergence points in phylogenies. The method joins a host of existing tests to detect selection sweeps along pre-specified branches of a phylogeny $[10,11,31,33,35-40,42,43]$. Unlike existing methods, however, the proposed method does not require a priori branch specification.

The method is developed in a Bayesian context and applied to two viral data sets. The HIV data strongly support the presence of at least one divergence point, while the HBV data fit a model with substantial site-to-site rate variation but no sudden or dramatic temporal rate shifts. Heterotachy may still occur in the HBV sequences, perhaps more subtly, accumulating slowly and methodically over time as in the covarion model [29]. The presence of a divergence point between HIV subtypes B and C indi- 


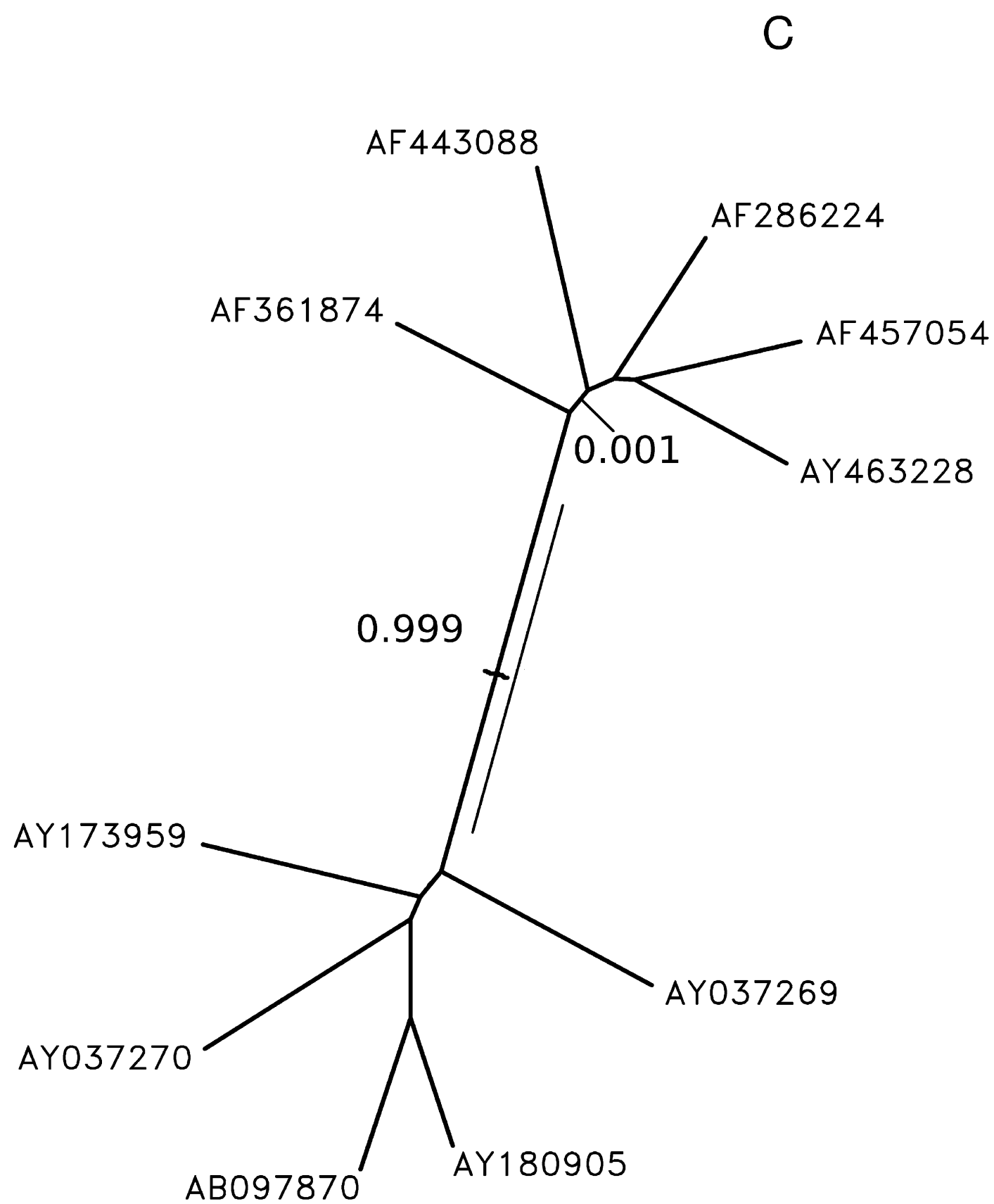

B

Figure 6

Phylogenetic tree of HIV subtypes B and C. Phylogenetic tree inferred from HIV data. The topology is not estimated, but branch lengths are shown at their posterior means. The posterior mean location of the divergence point is shown along with a parallel bar demarcating the $95 \%$ Bayesian credible interval. The numbers indicate the conditional posterior probability that the indicated branch carries the divergence point given there is a divergence point in the tree. For reference, the length of the middle branch is 0.12 . 
Table 3: HBV estimated parameters.

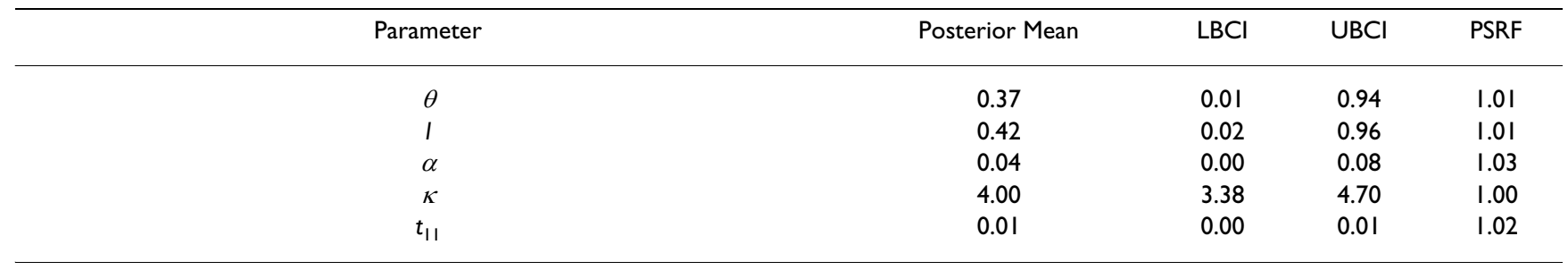

$\log _{10} B_{D P}=-0.64$ (substantial support against)

$\log _{10} B_{F H}=-0.71$ (substantial support against)

See the caption of Table 2. The second Bayes factor is the support for a divergence point along the middle branch separating the two genotypes.

cates a profound rate change after the split of these two subtypes. It does not prove that the divergence point occurred concurrent with the split or caused it in any way. However, it is plausible that host population differences are related to the apparent selection differences, and further testing may reveal specific cause-and-effect relationships between host differences and rate differences in the HIV genome. Finally, I make no effort to detect the possi-

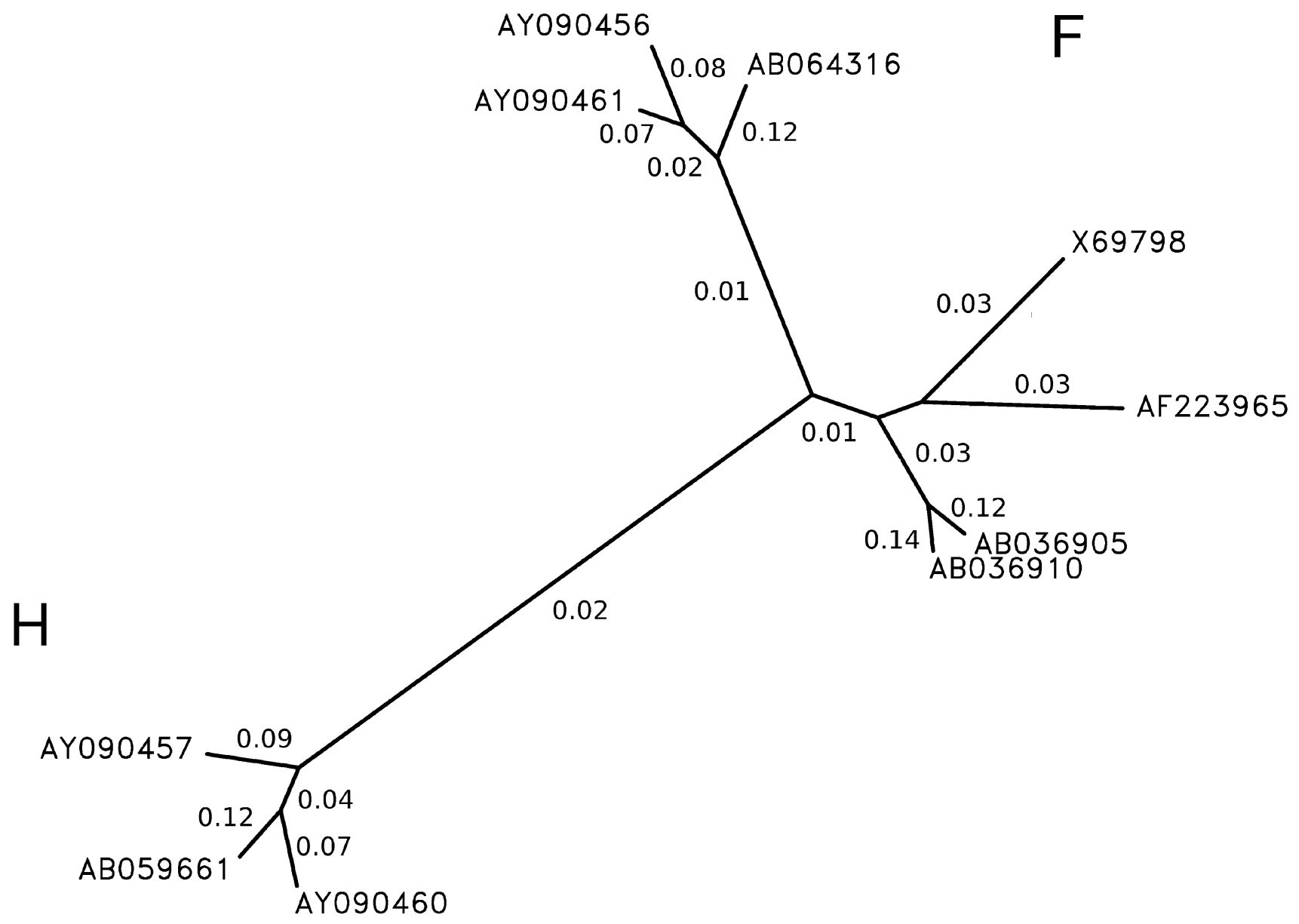

\section{Figure 7}

Phylogenetic tree of HBV subtypes F and H. Phylogenetic tree inferred from HBV data. The topology is not estimated, but branch lengths are shown at their posterior means. The numbers by each branch indicate the conditional posterior probability that the indicated branch carries the divergence point given a divergence point is present in the tree. In fact, there is substantial support against a divergence point in this data set. For reference, the length of the middle branch is 0.07 
Table 4: Proposal distributions.

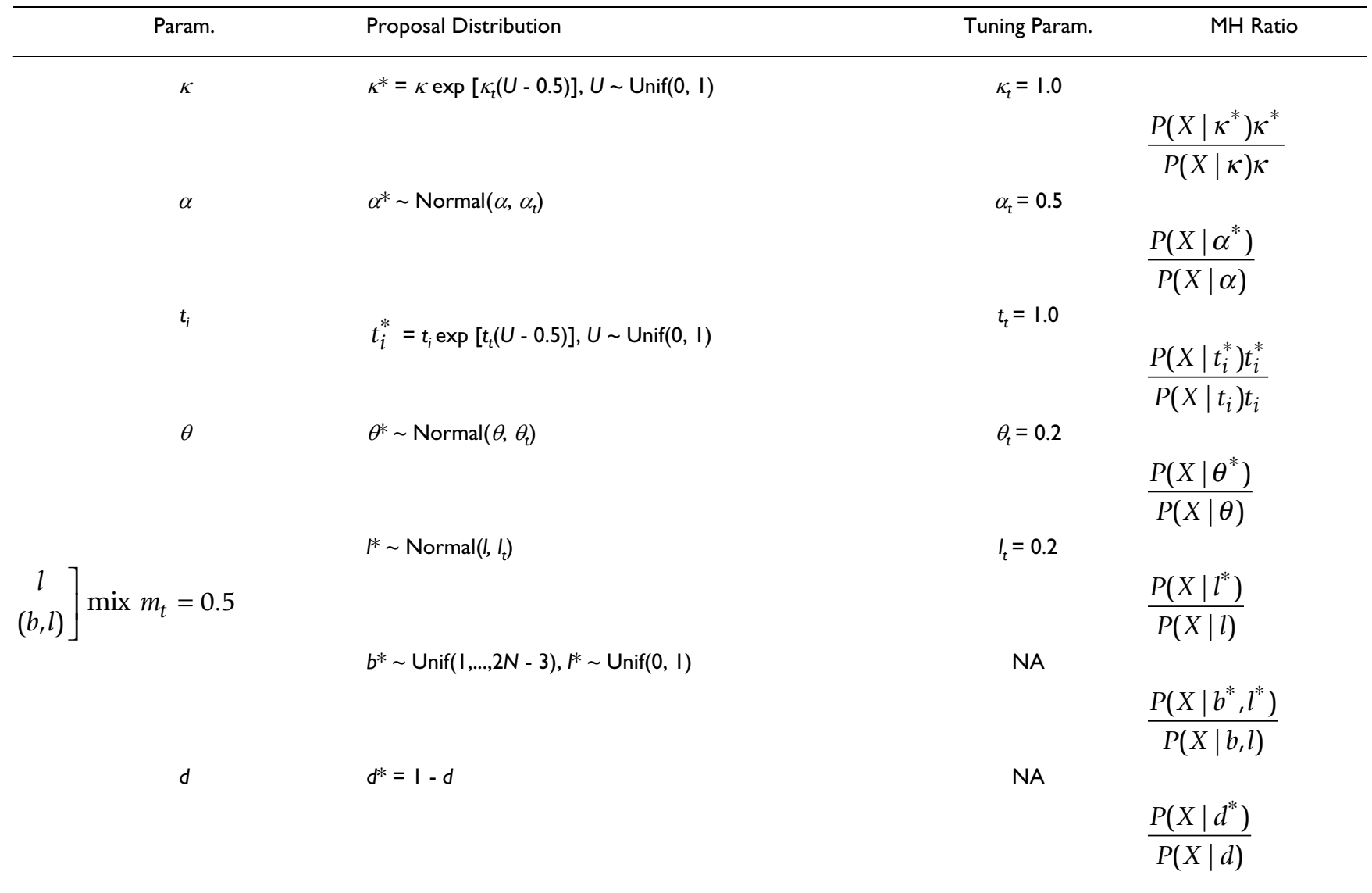

\footnotetext{
For each parameter listed, a new value (starred, e.g. $\kappa^{*}$ ) is proposed according to the listed distribution. Branch lengths $t_{i}$ are updated one at a time for $i=I, \ldots, 2 N-3$. Tuning parameters are subscripted by $t$. Updates of either $I$ or $(b, I)$ are mixed with probability $m_{t}$. Update of $d$ is a transdimensional move. The Metropolis-Hastings acceptance ratios are given in the last column. Dependence on parameters not involved in the update is not shown.
}

ble presence of other, weaker divergence points in this set of HIV data. A natural extension of the current Bayesian method is to allow and estimate more than one divergence point per phylogenetic tree. Interestingly, the episodic evolutionary events sought by methods that detect high nonsynonymous rates on particular branches $[42,43]$ consist of two divergence points appearing close to or on the same branch of a phylogeny. Thus, multi-divergence point models may be better equipped to detect and quantitate temporary shifts in evolution. In the limit as the number of divergence points $d$ increases and the proportion of diverging sites $\theta$ decreases, the model approaches the covarion model.

Recombination between subtypes/genotypes is a common phenomena in both HIV [66] and HBV [67] that can result in a non-constant topology or branch lengths along an alignment. Another oversight that could lead to nonconstant branch lengths along the alignment is the fact that both data sets consist of multiple genes. While site-tosite rate variation can account for some rate variation along the alignment, it does not adequately model wholegene shifts in rate that can result when different genes evolve according to different processes. In assuming that a fixed topology and a single set of branch lengths applies to the full alignment, I prohibit the possibility of recombination or gene effects and may force the wrong topology and or branch lengths on some sites if these assumptions are not met. Forcing either an incorrect topology or incorrect branch lengths could affect inference of heterotachy. The first set of simulations and Figure 2 demonstrate that branch length estimation can depend on the estimation of heterotachy and rate variation. The reverse must also be true, such that incorrect branch lengths and especially topology, could influence inference on $\theta, l$, and possibly even the presence of a divergence point. To limit the possibility of such an artifact, all selected viral sequences had been previously reported as nonrecombinant or verified so using recombination detection software [68]. This step insures that the two subtrees are consistent throughout the alignment, however it does not guarantee that the subtree topologies are consistent. Another natural extension 
of the current model is to include recombination models that allow topology and branch length variation along the alignment while simultaneously estimating heterotachy. Both models are already computationally difficult when considered separately, and combining them obviously represents a major computational challenge.

The current approach does not estimate the phylogenetic tree, assuming that it can be derived confidently using other methods. This assumption requires serious reconsideration since heterotachy can affect phylogenetic tree estimation $[13,69]$. In addition, including additional taxa should improve the power of the method to detect selection and estimate $\theta$, yet adding taxa increases the chance of topological uncertainty. The ideal solution is to simultaneously estimate phylogeny and divergence point location. Unfortunately, because branches do not retain definition across different topologies, divergence points may also lose definition. Presumably, however, a simultaneous estimation procedure should be able to detect strong divergence points on supported branches while allowing for topological uncertainty within clades.

A persistent question lurking behind these analyses is whether detected rate variation is actually connected to selection and function [70]. Lopez et al. [49] suggest that temporal rate variation need not relate to function because even mitochondrial cytochrome $b$, whose function is highly conserved among all vertebrates, tests positive for heterotachy. Comparison of vertebrate $\alpha$ and $\beta$ globins revealed a similar disconnect between significantly heterotachous sites and those sites most likely responsible for protein functional differences [71], leading Philippe et al. [72] to conclude heterotachy may be a largely neutral evolutionary process on alternative, but viable protein conformations. In order to identify functionally important rate shifts, it may be necessary to design models that separate this neutral heterotachy, e.g. covarion-like models, from the sudden and temporary heterotachy of the type expected after gene duplication or other environmental shifts. Yet even these models may be over-simpistic. Protein models $[73,74]$ that allow amino acid sites to self-classify into highly flexible evolutionary classes, reveal that sites with different functional or structural jobs differ not only in their evolutionary rates, but also in how they mutate. The divergence point described here allows no such changes in site properties. For example, if transitions are much more likely than transversions in one rate class, they are identically skewed in all other rate classes. Yet it is plausible that the skew will shrink at certain codon positions or within some amino acid contexts. Despite all these caveats, a recent large-scale analysis of proteins with known function reveals that shifts in rate are good predictors of differing functional classes [75].
Although the role of heterotachy in evolution remains to be clearly defined, the ability to detect rate variation from sequences alone is a powerful resource provided by comparative genomics. I did not use the results to predict functionally important sites in the viral data sets, but the forward-backward algorithm can compute the most likely unobserved state, diverged or not, of each nucleotide site [76]. In a Bayesian context, the prediction can be integrated against the MCMC-approximated posterior density to improve robustness. Specific predictions can generate hypotheses and focus future biological experiment.

\section{Methods \\ Model}

We start with an alignment $X$ of $N$ sequences of length $L$. Nucleotide $X_{i j} \in\{A, C, G, T, U,-\}$ is the nucleotide at position $j$ of sequence $i$, which may be a gap (-). Sites $X_{\text {. }}$ are treated as independent for all $j=1, \ldots, L$. The evolutionary rate at site $j$ is assumed to be selected at random from a discretized gamma distribution with four equi-probable rates and shape parameter $\alpha$ [25]. Site-to-site rate variation is greater for smaller $\alpha$, particularly for $\alpha<1$. Because the actual evolutionary rate of site $j$ is unknown, the likelihood of the site $j$ data is integrated against all possible rates, using the discrete gamma approximation and removing explicit dependence on the site-specific rate $r_{j}$. The site likelihood is

$P\left(X_{\cdot j} \mid \alpha, \tau, t, \kappa\right)=\sum_{r_{j}} P\left(X_{\cdot j} \mid \tau, r_{j}, t, \kappa\right) P\left(r_{j} \mid \alpha\right)$,

where $\tau$ is the unrooted topology, $t=\left(t_{1}, \ldots, t_{2 N-3}\right)$ are the branch lengths, $\kappa$ is the transition/transversion ratio assuming the HKY85 nucleotide substitution model [19], and $P\left(r_{j} \mid \alpha\right)=0.25$ is the probability of drawing rate $r_{j}$ from the discrete gamma distribution. Because sites are assumed independent, the full data likelihood is

$P(X \mid \alpha, \tau, t, \kappa)=\prod_{j=1}^{L} P\left(X_{\cdot j} \mid \alpha, \tau, t, \kappa\right)$.

The model just described is commonly referred to as the variable rates across sites or RAS model.

I now introduce the possibility of a divergence point (DP) into the model. Place a divergence point (DP) in the phylogenetic tree $(\tau, t)$ (Figure 1), on branch $b \in\{1, \ldots, 2 N-3\}$ at relative position $l \in[0,1]$ with respect to an arbitrary root. A fraction $\theta$ of sites randomly select a new rate after crossing the divergence point, so rate $r_{j 1}$ applies along all the branches in subtree $\tau_{1}$, including the stub of the branch dissected by the DP and $r_{j 2}$ applies throughout subtree $\tau_{2}$ along with the other part of the dissected branch. When there is a divergence point, the full site likelihood is 
$P\left(X_{\cdot j} \mid \alpha, \tau, t, \kappa\right)=\theta \sum_{r_{11}, r_{i 2}} P\left(X_{(1) j} \mid \tau_{1}, r_{j 1}, t, \kappa\right) P\left(X_{(2) j} \mid \tau_{2}, r_{j 2}, \kappa\right) P\left(r_{j 1}, r_{j 2} \mid \alpha\right)+(1-\theta) \sum_{r_{j}} P\left(X_{\cdot j} \mid \tau, r_{j}, t, \kappa\right) P\left(r_{j} \mid \alpha\right)$,

where $X_{(1) j}$ are the site $j$ data for sequences of subtree 1 and $X_{(2) j}$ are the data for the sequences of subtree 2 . The first term is the likelihood of the site $j$ data if the site diverges across the DP, with data $X_{(1) j}$ produced by an evolutionary process with rate $r_{j 1}$ and data $X_{(2) j}$ produced by a process with rate $r_{j 2}$. The second term is the likelihood of site $j$ if there is no divergence across the DP and both data $X_{(1) j}$ and $X_{(2) j}$, i.e. the full site data $X_{. j}$ evolve with common rate $r_{j}$. Again, whether site $j$ diverges across the DP is not known, so the full site likelihood is obtained by integrating against the Bernoulli probability distribution for divergence at the DP. If there is no divergence point in the phylogenetic tree, then $\theta=0$ and the site likelihood is given by equation (1) under the RAS model.

\section{Prior Distributions}

Because the model is implemented in a Bayesian context, a prior distribution must be specified for all model parameters. Table 5 lists all prior distributions. Uninformative or nearly uninformative priors are used for most model parameters. The continuous parameters, transition/transversion ratio $\kappa \in[0, \infty)$, branch lengths $t_{\kappa} \in[0, \infty), \kappa=$ $1, \ldots, 2 \mathrm{~N}-3$, and discrete gamma shape parameter $\alpha \in[0$, $\infty)$ are assumed uniform over a wide range well beyond biologically relevant limits. The DP location $l \in[0,1]$ is uniform throughout its range. The discrete parameter $b$, indicating the branch of the DP, is uniform over all possible branches. We assume the topology $\tau$ is known and do not estimate it using the model.

In addition, we place a prior on the presence or absence of a divergence point somewhere in the phylogenetic tree. Let $d$ be the number of divergence points in the tree. With probability $P(d=1)=0.5$ there is a single divergence point at location $(b, l)$. Otherwise and also with probability $P(d$ $=0)=0.5$ there is no divergence point and the RAS model applies. In fact, it may be biologically possible for more than one divergence point to coexist in a phylogenetic tree. We do not consider these more complex models by setting $P(d>1)=0$.

\section{MCMC}

The posterior distribution is estimated via MCMC using Metropolis-Hastings (MH) within Gibbs sampling. Supplemented with $d$, the parameter vector is now either $(1$, $\kappa, \alpha, t, \theta, l, b)$ when there is a divergence point or $(0, \kappa, \alpha$, $t$ ) in the absence of a divergence point. Clearly, the dimension of the parameter space changes with $d$ and there are two types of MCMC updates, those within a fixed dimension and trans-dimensional moves. For fixed dimensional updates, the following sequence of moves is applied within a Gibbs cycle to update from $\left(\kappa_{n^{\prime}} \alpha_{n^{\prime}} t_{n^{\prime}} \theta_{n^{\prime}} l_{n^{\prime}} b_{n}\right)$ to $\left(\kappa_{n+1}, \alpha_{n+1}, t_{n+1}, \theta_{n+1}, l_{n+1}, b_{n+1}\right)$ via incremental proposals of $\left(\kappa^{*}, \alpha^{*}, t^{*}, \theta^{*}, l^{*}, b^{*}\right)$.

$$
\begin{aligned}
& \kappa^{*} \mid \kappa_{n^{\prime}} \alpha_{n^{\prime}} t_{n^{\prime}} \theta_{n^{\prime}} l_{n^{\prime}} b_{n}=\kappa_{n} e^{(U-0.5)}, U \sim \operatorname{Unif}(0,1) \\
& \alpha^{*} \mid \kappa_{n+1}, \alpha_{n^{\prime}} t_{n^{\prime}}, \theta_{n^{\prime}} l_{n^{\prime}}, b_{n}=\operatorname{Normal}\left(\alpha_{n^{\prime}} 0.5\right)
\end{aligned}
$$

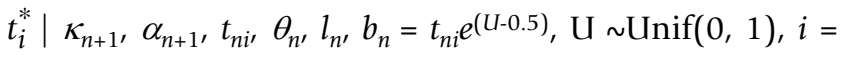

$$
\begin{aligned}
& 1, \ldots, 2 \mathrm{~N}-3 \\
& \theta^{*} \mid \kappa_{n+1}, \alpha_{n+1}, t_{n+1}, \theta_{n^{\prime}} l_{n^{\prime}} b_{n} \sim \operatorname{Normal}\left(\theta_{n^{\prime}} 0.2\right) \\
& l^{*} \mid \kappa_{n+1}, \alpha_{n+1}, t_{n+1}, \theta_{n+1}, l_{n^{\prime}} b_{n} \sim \operatorname{Normal}\left(l_{n^{\prime}} 0.2\right) \\
& b^{*}, l^{*} \mid \kappa_{n+1}, a_{n+1}, t_{n+1}, \theta_{n+1}, l_{n}, b_{n} \text { via } b^{*} \sim \text { Uniform }(1, \ldots, b \text { - } \\
& 1, b+1, \ldots, 2 N-3), l^{*} \sim \operatorname{Uniform}(0,1) \text { independently. }
\end{aligned}
$$

Here $t_{n i}$ is the $i$ th branch length listed in vector $t_{n}$ of the $n$th MCMC sample. Following Minin et al. [68], most parameters defined on the positive real line are updated using an exponential updater that proposes large changes when the parameter is large and small changes when the parameter is small. The DP location, $\theta$, and $\alpha$ are updated using a reflected normal updater. A large variance is applied because posterior variances tend to be large. To update the branch $b$ containing the DP, a joint move is used that proposes a new branch $b^{*}$ uniformly from among all but the current DP branch and proposes a new $l^{*}$ uniformly from the prior Unif $(0,1)$. During each cycle, only one of the last two moves, either updating $l$ separately or $(b, l)$ jointly, is attempted according to a user-specified mixing probability. For all MCMC samples, this mixing parameter was set to 0.5 .

I use reversible jump MCMC [77] to carry out transdimensional moves. When proposing to increase $d$ from zero to one, the parameter space of the model is supplemented by drawing random variables $z_{b}, z_{l}$ and $z_{\theta}$ independently from the prior distributions of $b, l$, and $\theta$, respectively. The one-to-one transformation across dimensions is

$$
\begin{aligned}
& d^{*}=1-d \\
& \theta^{*}=z_{\theta} \\
& \kappa^{*}=\kappa \\
& l^{*}=z_{l} \\
& \alpha^{*}=\alpha \\
& b^{*}=z_{b}
\end{aligned}
$$


Table 5: Prior distributions

\begin{tabular}{cl}
\hline Parameter & Distribution \\
\hline$\kappa$ & Unif $(0,1000)$ \\
$\alpha$ & Unif $(0,100)$ \\
$t_{i}$ & Unif $(0,100)$, for $i=1, \ldots, 2 N-3$ \\
$\theta$ & Unif $(0,1)$ \\
$l$ & Unif $(0, I)$ \\
$b$ & Unif $(I, \ldots, 2 N-3)$ \\
$\tau$ & Not estimated \\
$d$ & $P(d=0)=P(d=1)=0.5$ \\
\hline
\end{tabular}

$t_{i}^{*}=t_{i}$ for all $i$,

which has a Jacobian determinant of one. A trans-dimensional move is attempted during every Gibbs cycle.

Most proposal distributions can be tuned with userdefined tuning parameters. Tuning parameter values used to compute all results in this report are listed in Table 4 along with a summary of all proposal distributions, including Metropolis-Hastings $(\mathrm{MH})$ acceptance ratios. All Metropolis-Hastings acceptance ratios reduce to simple expressions of the likelihoods under the current and proposed states.

The specified model and MCMC algorithm is implemented in a computer program written in $\mathrm{C}$ and available upon request from the author.

\section{Hypothesis Testing}

To estimate error rates and power, I compute the Bayes factor [78] in favor of the hypothesis of a divergence point somewhere in the phylogeny vs. the hypothesis of the RAS model without a divergence point. Due to the chosen prior on the divergence point indicator $d$, this Bayes factor is particularly simple

$B_{D P}=\frac{P(d=1 \mid X)}{P(d=0 \mid X)} \approx \frac{\# \text { samples with DP }}{\# \text { samples without DP }}$.

Following [78], $\log _{10} B_{D P}>1$ is taken to indicate strong support for a divergence point. Conversely, $\log _{10} B_{D P}<-1$ lends strong support to the absence of a divergence point. For Bayes factor falling in the region of ambiguity between -1 and 1 , no decision can be made with confidence.

It is also possible to compute Bayes factors to test the presence of divergence points on particular branches. Specifically, the Bayes factor in favor of a divergence point along branch $j$ is

$$
B_{j}=\frac{P(b=j \mid X) P(b \neq j)}{P(b=j) P(b \neq j \mid X)}
$$

where $b$ is the branch with the divergence point and $P(b=$ $j$ ) is the prior probability of a divergence point along branch $j$ in the simulation topology. Because each branch is equally likely to carry the divergence point $a$ priori, $P(b$ $=j)=\frac{1}{2 N-3}$ for all $j$. I compute $B_{j}$ when $B_{D P}>1$ or regardless of $B_{D P}$ in the case of the HBV data. For the viral data sets I denote $B_{B C}$ and $B_{F H}$ to be the Bayes factors for the branches separating particular named subtrees.

\section{Simulation}

I design a simulation study to verify the code and examine the sensitivity of the method. All sequences are evolved assuming the divergence model described above and assuming the HKY85 [19] model of evolution. I vary $\alpha \in$ $\{0.01,0.1,0.5,1.0,2.0\}$ and $\theta \in\{0.0,0.1,0.3,0.5,0.7$, $0.9\}$ to produce a grid of simulation conditions. Note, that the condition $\theta=0$ implies no divergence point. I then simulate 100 datasets for each combination of $(\alpha, \theta)$ assuming the topology of Figure 1, with a DP located at position $l=0.9$ on the middle branch. All simulated data sets are 1,000 nucleotides long and all branch lengths are fixed at 0.1 . At the root, each simulated site is assigned a rate, from a choice of 4 possibilities obtained via equiprobable discretization of the gamma distribution [25]. At the DP, a site is induced to select a new rate class with probability $\theta$. Sites that experience a selection shift, i.e. they select a new rate class at the DP, may choose the same rate class with probability 0.25 .

I also carry out a number of other simulations. I start by simulating data sets while simultaneously varying $L \in$ $\{1000,2500,5000,7500\}, t_{j} \in\{0.03,0.05,0.07,0.09\}, \alpha$ $\in\{0.7,0.5,0.3,0.1\}$, and $\theta \in\{0.1,0.3,0.5,0.7\}$. Each simulation condition is replicated only 10 times and are not shown. They are used principally to select conditions for other more extensive simulations. For example for Figure 4, I simulate 100 data sets of varying length $L=1000$, 2500,5000 , or 7500 and with varying branch length $t_{j}=$ $0.03,0.05,0.07$, or 0.09 for all branches $j$. The other parameters are fixed at $\alpha=0.7, \theta=0.5$, and $l=0.9$. For Figure 5, I simulate 40 data sets either with the divergence point on a terminal branch or on the middle branch, but with varying numbers of taxa, either $2,4,6$, or 8 , in each subtree. When the divergence point is on a terminal branch, the other parameters are $\alpha=\theta=0.5$, all branch lengths $t_{j}=0.1$, and alignment length is $L=1000$. When varying the number of taxa, the other parameters are $\alpha=$ $\theta=t_{j}=0.1$ and the alignment length $L=1000$.

Each simulated data set is examined in a single MCMC run of length 6000, burnin 1000, and subsample rate 5 . To assess whether this MCMC length, burnin, and subsample are sufficient for convergence, I randomly select 
one simulated data set per simulation condition and compute a second MCMC sample starting from a distinct initial state. For each pair of MCMC samples, I compute the potential scale reduction factor (PSRF) [54] for the log likelihood and parameters $\alpha, \kappa$, and all branch lengths $t_{j^{\prime}}$ as well as $\theta$ and $l$ wherever the latter are applicable. The PSRF statistic compares the between sample variance to within sample variance and should be near 1 . Of 150 PSRF statistics computed, 2 are above 1.1 and 15 are above 1.01. In addition, I perform a test of proportions for the posterior support of a divergence point, and found 2 significant results at the level of 0.05 , for a error rate of about 0.07 . In neither case, did the classification of the sample as supporting heterotachy or homotachy differ between the samples.

\section{Comparison to existing methods}

To compare the proposed method with existing methods for detecting temporal rate variation at specific branches, I implement two techniques and apply them to the simulated data. Both techniques rely on specifying two groups of sequences $a$ priori. Naturally, the groups I utilize are 0 , 1, 2, 3 from subtree 1 and 4, 5, 6, 7 from subtree 2 in Figure 1 .

Ané et al. [33] propose to compare two groups by comparing the proportions of invariable sites. Their test statistic is

$$
W=\frac{L_{12}}{L}-\frac{L_{1} L_{2}}{L^{2}}
$$

where $L_{12}$ is the number of sites that vary in both subtrees, $L_{1}$ is the number of sites that vary in subtree 1 , and $L_{2}$ is the number of sites that vary in subtree 2 . When the two subtrees are completely independent, $W=0$, however because of a shared ancestor nucleotide and site-to-site rate variation, $W$ will usually exceed zero. The presence of a divergence point on the branch separating the two subtrees will decrease $W$ by increasing the independence of rates between the two subtrees. Parametric bootstrapping is used to determine whether $W$ is significantly smaller than would be expected given statistical variation under the RAS model. For each simulated data set, I estimate $\alpha$, $\kappa$, stationary nucleotide frequencies $\pi$ and branch lengths $t$ using PHYML [79]. I do not estimate, rather assume the true topology. Seq-Gen [80] generates 100 parametric bootstrap datasets under the RAS model using the PHYML-generated parameter estimates, and the $W$ statistic is computed for each. The proportion of bootstrap replicates whose statistic falls below the $W$ observed for the original simulated dataset is the p-value for rejecting the RAS model.

Lopez et al. [13] describe another test for comparing not just the invariant sites, but the distribution of mutations at all variable sites between two subtrees. One first estimates the number of mutations in each subtree, using the method of Gu and Zhang [81]. Because the Gu and Zhang method returns non-integer estimates of the number of mutations within the subtrees, I round these numbers to the nearest integer before preceeding. The distribution of mutations across sites is then compared between the two subtrees using a chi-square statistic for a $2 \times L$ table. Since the asymptotic properties of the chi-square distribution generally do not apply to such data, significance is assessed by 100 permutations of the data while keeping the total number of mutations at each site and within each group (i.e. row and column totals) constant.

\section{Viral data sets}

I collect 5 subtype B [GenBank:AB097870, AY037269,

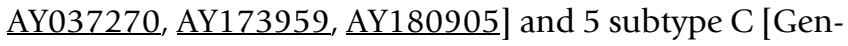
Bank:AF286224， AF457054， AF361874， AF443088, AY463228] sequences from the HIV database [82] and align them using clustalW [83]. The final alignment is 6610 base pairs long and represents $68 \%$ of the entire HIV genome. I collect 7 subtype F [GenBank:AB036905, $\underline{\mathrm{AB} 036910}, \underline{\mathrm{AB} 064316}, \underline{\mathrm{AF} 223965}, \underline{\mathrm{AY} 090456}, \underline{\mathrm{AY} 090461}$, X69798] and 3 subtype $H$ [GenBank:AB059661, AY090457, AY090460] sequences from GenBank and align them using clustalW [83]. The final alignment is 3215 base pairs long and represents the entire HBV genome. For each viral data set, I produce $6 \mathrm{MCMC}$ samples of size 1000 from a run of length 6000, burnin 1000, and subsample rate 5 . To assess convergence, I compute PSRF [54] of all parameters $\theta, \alpha, \kappa, b, t, l$.

\section{Acknowledgements}

The author is indebted to the reviewers and editor for very extensive and thoughtful comments that greatly improved the manuscript. This work was partially supported by grant GM068955. The calculations in this work were performed in part on the cluster made possible by the support of NSFIGERT, the Plant Sciences Institute, and the Office of the Provost at lowa State University.

This article has been published as part of BMC Evolutionary Biology Volume 7 Supplement I, 2007: First International Conference on Phylogenomics. The full contents of the supplement are available online at http:// www.biomedcentral.com/bmcevolbiol/7? issue=SI.

\section{References}

I. Frost SDW, Gunthard HF, Wong JK, Havlir D, Richman DD, Leigh Brown $\mathrm{A}$ : : Evidence for positive selection driving the evolution of HIV-I env under potent antiviral therapy. Virology 2001 , 284:250-258.

2. Chen SL, Hung CS, Xu J, Reigstad CS, Magrin V, Sabo A, Blasiar D, Bier T, Meyer RR, Ozersky P, Armstrong JR, Fulton RS, Latreille JP, Speith J, Hooton TM, Mardis ER, Hultgreen SJ, Gordon JI: Identification of genes subject to positive selection in uropathogenic strains of Escherichia coli: A comparative genomics approach. Proc Natl Acad Sci USA 2006, I 03:5977-5982.

3. Yang Z: Likelihood ratio tests for detecting positive selection and application to primate lysozyme evolution. Mol Biol Evol 1998, I 5:568-573.

4. Arbiza L, Duchi S, Montaner D, Burguet J, Pantoja-Uceda D, PinedaLucena A, Dopazo J, Dopazo H: Selective pressures at a codon- 
level predict deleterious mutations in human disease genes. J Mol Biol 2006, 358: | 390-|404.

5. Crowe ML, Wang XQ, Rothnagel JA: Evidence for conservation and selection of upstream open reading frames suggests probable encoding of bioactive peptides. BMC Genomics 2006 7:16.

6. Mekel-Bobrov N, Gilbert SL, Evans PD, Vallender EJ, Anderson JR, Hudson RR, Tishkoff SA, Lahn BT: Ongoing adaptive evolution of ASPM, a brain size determinant in Homo sapiens. Science 2005, 309: 1720-1722.

7. Jobson RW, Nielsen R, Laakkonen L, Wikstrom M, Albert VA: Adaptive evolution of cytochrome c oxidase: Infrastructure for a carnivorous plant radiation. Proc Natl Acad Sci USA 2004 | 0 | : | 8064- |8068.

8. Gaucher EA, Miyamoto MM, Benner SA: Function-structure analysis of proteins using covarion-based evolutionary approaches: Elongation factors. Proc Natl Acad Sci USA $200 \mathrm{I}$, 98:548-552

9. Wang $\mathrm{Y}, \mathrm{Gu} X$ : Functional divergence in the caspase gene family and altered functional constrains: statistical analysis and prediction. Genetics 200I, I 58: | 3 II-I320.

10. Knudsen B, Miyamoto MM: A likelihood ratio test of evolutionary rate shifts and functional divergence among proteins. Proc Natl Acad Sci USA 200I, 98: I45। 2-145I7.

II. Susko E, Inagaki Y, Field C, Holder ME, Roger AJ: Testing for differences in rates-across-sites distributions in phylogenetic subtrees. Mol Biol Evol 2002, I 9: | 5 |4-1523.

12. Inagaki Y, Susko E, Fast NM, Roger AJ: Covarion shifts cause a long-branch attraction artifact that unites microsporidia and archaebacteria in EF-I $\alpha$ phylogenies. Mol Biol Evol 2004, 2 I:|340-1349.

13. Lopez $\mathrm{P}$, Forterre $\mathrm{P}$, Philippe $\mathrm{H}$ : The root of the tree of life in the light of the covarion model. J Mol Evol 1999, 49:496-508.

14. Fitch WM, Margoliash E: A method for estimating the number of invariant amino acid coding positions in a gene using cytochrome c as a model case. Biochem Genet 1967, I:65-71.

15. Fitch WM: The estimate of total nucleotide substitutions from pairwise differences is biased. Philos Trans $R$ Soc Lond B Biol Sci 1986, 3 I 2:317-324.

16. Uzzell T, Corbin KW: Fitting discrete probability distributions to evolutionary events. Science 1971, I 72:1089-1096.

17. Chang JT: Inconsistency of evolutionary tree topology reconstruction methods when substitution rates vary across characters. Math Biosci 1996, I 34: I89-2I5.

18. Gaut BS, Lewis PO: Success of maximum likelihood phylogeny inference in the four-taxon case. Mol Biol Evol I995, I 2: I52-I62.

19. Hasegawa M, Kishino H, Yano T: Dating the human-ape split by a molecular clock of mitochondrial DNA. I Mol Evol 1985, 22:160-192.

20. Golding GB: Estimates of DNA and protein sequence divergence: an examination of some assumptions. Mol Biol Evol 1983, I:I25-142

21. Jin L, Nei M: Limitations of the evolutionary parsimony method of phylogenetic analysis. Mol Biol Evol 1990, 7:82-102.

22. Yang Z: Maximum-likelihood estimation of phylogeny from DNA sequences when substitution rates differ over sites. Mol Biol Evol 1993, I0:1396-1401.

23. Waddell PJ, Steel MA: General time-reversible distances with unequal rates across sites: Mixing $\Gamma$ and inverse Gaussian distributions with invariant sites. Mol Phylogenet Evol 1997, 8:398-414

24. Pond SK, Muse SV: Site-to-site variation of synonymous substitution rates. Mol Biol Evol 2005, 22:2375-2385.

25. Yang Z: Maximum likelihood phylogenetic estimation from DNA sequences with variable rates over sites: approximate methods. J Mol Evol 1994, 39:306-314.

26. Fitch WM: Further improvements in the method of testing for evolutionary homology among proteins. J Mol Biol 1970, 49:1-14.

27. Fitch WM, Markowitz E: An improved method for determining codon variability in a gene and its application to the rate of fixation of mutations in evolution. Biochem Genet 1970, 4:579-593.

28. Shoemaker JS, Fitch WM: Evidence from nuclear sequences that invariable sites should be considered when sequence divergence is calculated. Mol Biol Evol 1989, 6:270-289.
29. Tuffley C, Steel M: Modeling the covarion hypothesis of nucleotide substitution. Math Biosci 1998, |47:63-9|.

30. Galtier N: Maximum-likelihood phylogenetic analysis under a covarion-like model. Mol Biol Evol 200I, I 8:866-873.

31. Huelsenbeck JP: Testing a covariotide model of DNA substitution. Mol Biol Evol 2002, I 9:698-707.

32. Galtier N, Jean-Marie A: Markov-modulated Markov chains and the covarion process of molecular evolution. J Comput Biol 2004, I I:727-733.

33. Ane C, Burleigh JG, McMahon MM, Sanderson MJ: Covarion structure in plastid genome evolution: a new statistical test. Mo Biol Evol 2005, 22:9|4-924.

34. Smedmark JE, Swenson U, Anderberg AA: Accounting for variation of substitution rates through time in Bayesian phylogeny reconstruction of Sapotoideae (Sapotaceae). Mol Phylogenet Evol 2006, 39:706-72I.

35. Gu X: Statistical methods for testing functional divergence after gene duplication. Mol Biol Evol 1999, I6: | 664- I674.

36. Gu X: Maximum-likelihood approach for gene family evolution under functional divergence. Mol Biol Evol 200I, I 8:453-464.

37. Lockhart PJ, Steel MA, Barbrook AC, Huson DH, Charleston MA Howe Cl: A covariotide model explains apparent phylogenetic structure of oxygenic photosynthetic lineages. Mol Biol Evol 1998, 15: I 183-1 I88.

38. Lockhart PJ, Huson D, Maier U, Fraunholz MJ, Van De Peer Y, Barbrook AC, Howe CJ, Steel MA: How molecules evolve in eubacteria. Mol Biol Evol 2000, 1 7:835-838.

39. Miyamoto MM, Fitch WM: Testing the covarion hypothesis of molecular evolution. Mol Biol Evol 1995, I 2:503-5I3.

40. Pupko T, Galtier N: A covarion-based method for detecting molecular adaptation: application to the evolution of primate mitochondrial genomes. Proc Biol Sci 2002, 269: | 3 | 3- I 3 | 6 .

4I. Messier W, Stewart CB: Episodic adaptive evolution of primate lysozymes. Nature 1997, 385: I5 I-I54

42. Yang Z, Nielsen R: Codon-substitution models for detecting molecular adaptation at individual sites along specific lineages. Mol Biol Evol 2002, I 9:908-917

43. Zhang J, Nielsen R, Yang Z: Evaluation of an improved branchsite likelihood method for detecting positive selection at the molecular level. Mol Biol Evol 2005, 22:2472-2479.

44. Liberles DA, Schreiber DR, Govindarajan S, Chamberlin SG, Benner SA: The adaptive evolution database (TAED). Genome Biol 200I, 2:RESEARCH0028-.

45. Abhiman S, Sonnhammer EL: FunShift: a database of function shift analysis on protein subfamilies. Nucleic Acids Res 2005, 33:D197-D200

46. Yang Z: Among-site rate variation and its impact on phylogenetic analyses. Trends Ecol Evol 1996, I I:367-372.

47. Excoffier L, Yang Z: Substitution rate variation among sites in mitochondrial hypervariable region I of humans and chimpanzees. Mol Biol Evol 1999, I 6:1357-1368.

48. Baele G, Raes J, Van de Peer Y, Vansteelandt S: An improved statistical method for detecting heterotachy in nucleotide sequences. Mol Biol Evol 2006, 23:I397-I405.

49. Lopez $\mathrm{P}$, Casane D, Philippe H: Heterotachy, an important process of protein evolution. Mol Biol Evol 2002, I 9:1-7.

50. McCutchan FE: Understanding the genetic diversity of HIV-I. AIDS 2000, I4:S3I-S44.

51. HIV Sequence Database - Geography Tool [http:www.hiv.lanl.gov/components/hiv-db/new geography/geogra phy.comp

52. Perrin L, Kaiser L, Yerly S: Travel and the spread of HIV-I genetic variants. Lancet Infect Dis 2003, 3:22-27.

53. Moore CB, John M, James IR, Christiansen FT, Witt CS, Mallal SA: Evidence of HIV-I adapatation to HLA-restricted immune responses at a population level. Science 2002, 296:|439-|443.

54. Gelman A, Rubin DB: Inference from iterative simulation using multiple sequences. Stat Sci 1992, 7:457-472.

55. Bartholomeusz A, Schaefer S: Hepatitis B virus genotypes: com parison of genotyping methods. Rev Med Virol 2004, I 4:3-I 6

56. Robertson BH, Margolis HS: Primate hepatitis B viruses genetic diversity, geography and evolution. Rev Med Virol 2002, I2:|33-|4| 
57. Campos RH, Mbayed VA, Pineiro Y, Leone FG: Molecular epidemiology of hepatitis B virus in Latin America. J Clin Virol 2005 34:S8-SI3.

58. Guettouche T, Hnatyszyn HJ: Chronic hepatitis B and viral genotype: the clinical significance of determining HBV genotypes. Antivir Ther 2005, 10:593-604.

59. Schaefer S: Hepatitis B virus: significance of genotypes. J Viral Hepat 2005, I2:III-124.

60. Arauz-Ruiz P, Norder H, Robertson BH, Magnius LO: Genotype H a new Amerindian genotype of hepatitis $B$ virus revealed in Central America. J Gen Virol 2002, 83:2059-2073.

61. Kato H, Fujiwara K, Gish RG, Sakugawa H, Yoshizawa H, Sugauchi $F$ Orito E, Ueda R, Tanaka Y, Kato T, Miyakawa Y, Mizokami M: Classifying genotype $F$ of hepatitis $B$ virus into $F I$ and $F 2$ subtypes. World J Gastroenterol 2005, I I:6295-6304.

62. Penny D, McComish BJ, Charleston MA, Hendy MD: Mathematical elegance with biochemical realism: the covarion model of molecular evolution. I Mol Evol 200 I, 53:7 II-723.

63. Seeger G, Mason WS: Hepatitis B virus biology. Microbiol Mol Biol Rev 2000, 64:5I-68.

64. Lunter G, Ponting CP, Hein J: Genome-wide identification of human functional DNA using a neutral indel model. PloS Comput Biol 2006, 2:e5

65. Siepel A, Bejerano G, Pederson JS, Hinrichs AS, Hou M, Rosenbloom K, Clawson H, Spieth J, Hillier LW, Richards S, Weinstock GM, Wilson RK, Gibbs RA, Kent WJ, Miller W, Haussler D: Evolutionarily conserved elements in vertebrate, insect, worm, and yeast genomes. Genome Res 2005, 15:1034-1050.

66. Robertson DL, Sharp PM, McCutchan FE, Hahn BH: Recombination in HIV-I. Nature 1995, 374: I24-I26.

67. Bowyer SM, Sim JG: Relationships within and between genotypes of hepatitis B virus at points across the genome: footprints of recombination in certain isolates. J Gen Virol 2000 , 8I:379-392.

68. Minin VN, Dorman KS, Fang F, Suchard MA: Dual multiple changepoint model leads to more accurate recombination detection. Bioinformatics 2005, 21:3034-3042.

69. Philippe H, Zhou Y, Brinkmann H, Rodrigue N, Delsue F: Heterotachy and long-branch attraction in phylogenetics. BMC Evol Biol 2005, 5:50.

70. Soyer OS, Goldstein RA: Predicting functional sites in proteins: site-specific evolutionary models and their application to neurotransmitter transporters. J Mol Biol 2004, 339:227-242.

7I. Gribaldo S, Casane D, Lopez P, Philippe H: Functional divergence prediction from evolutionary analysis: A case study of vertebrate hemoglobin. Mol Biol Evol 2003, 20: 1754- 1759.

72. Philipe H, Casane D, Gribaldo S, Lopez P, Meunier J: Heterotachy and functional shift in protein evolution. IUBMB Life 2003, 55:257-265.

73. Dimmic MW, Mindell DP, Goldstein RA: Modeling evolution at the protein level using an adjustable amino acid fitness model. Pac Symp Biocomput 2000, 5: 18-29.

74. Soyer OS, Dimmic MW, Neubig RR, Goldstein RA: Dimerization in aminergic G-protein-coupled receptors: application of a hidden-site class model of evolution. Biochemistry 2003, 42:|4522-|453|.

75. Abhiman S, Sonnhammer ELL: Large-scale prediction of function shift in protein families with a focus on enzymatic function. Proteins 2005, 60:758-768.

76. Rabiner LR, Juang $B H$ : An introduction to hidden markov models. IEEE ASSP Mag 1986, 3:4-16 [http://ieeexplore.ieee.org/xpls/ abs all.jsp?arnumber $=\mid 165342]$.

77. Green P: Reversible jump Markov chain Monte Carlo computation and Bayesian model determination. Biometrica 1995, 82:7II-732.

78. Kass RE, Raftery AE: Bayes Factors. JASA 1995, 90:773-795.

79. Guindon S, Gascuel O: A simple, fast, and accurate algorithm to estimate large phylogenies by maximum likelihood. Syst Biol 2003, 52:696-704.

80. Rambaut A, Grassly NC: Seq-Gen: an application for the Monte Carlo simulation of DNA sequence evolution along phylogenetic trees. Comput Appl Biosci 1997, 13:235-238.

81. Gu X, Zhang J: A simple method for estimating the parameter of substitution rate variation among sites. Mol Biol Evol 1997 14:1106-1113.
82. HIV Sequence Database [http://hiv-web.lanl.gov/content/hiv-db/ mainpage.html]

83. Thompson JD, Higgins DG, Gibson TJ: CLUSTAL W: improving the sensitivity of progressive multiple sequence alignments through sequence weighting, position specific gap penalties and weight matrix choice. Nucl Acids Res 1994, 22:4673-4680.
Publish with Biomed Central and every scientist can read your work free of charge

"BioMed Central will be the most significant development for disseminating the results of biomedical research in our lifetime. "

Sir Paul Nurse, Cancer Research UK

Your research papers will be:

- available free of charge to the entire biomedical community

- peer reviewed and published immediately upon acceptance

- cited in PubMed and archived on PubMed Central

- yours - you keep the copyright
BioMedcentral 